\title{
RÉFLEXIONS SUR LA CONSTRUCTION DU PONT DU GARD
}

\author{
Jean-Louis PAILLET
}

\begin{abstract}
Mots-clés. Narbonnaise, Nîmes, Pont du Gard, architecture, bassin de régulation, échafaudages, économie générale du chantier; engins de levage, inscription, matériaux et techriques de construction, hydraulique antique.

Résumé. Les récentes recherches conduites sur l'aqueduc de Nîmes permettent une nouvelle approche de la conception du Pont du Gard et des techniques mises en ceuvre au cours du chantier de sa construction : adaptation au relief et choix du site du franchissement du Gardon, conséquences de la pente variable du canal, statique et déformation de l'ouvrage, approvisionnement du chantier en pierre de taille, en chaux, en sable, en bois et métaux, nombre et qualification de la main-d'auvre nécessaire.

Cette nouvelle lecture de la construction du Pont du Gard est aussi l'occasion de proposer de nouvelles hypothèses sur les techniques de sa mise en cuvre fondées sur des observations réalisées sur le monument lui-même, sur des comparaisons et sur un raisonnement logique guidé par un double souci d'efficacilé et d'économie.
\end{abstract}

Key-words. Narbonensis, Nimes, Pont du Gard, architecture, regulation tank, scaffolding, management of the working site, lifting appliance, inscription, building material and techniques, hydraulics.

Abstract. Recent research on the aqueduct of Nimes allows a new approach of the Pont du Gard, planning and techniques selected during its construction: adaptation to the relief and choice of the crossing site of the river Gardon, due to the variable slope of the channel, static and distortion of the structure, supply in stone, lime, sand, wood and metal, staff needed (number and qualification).

This new reading of the construction gives also the opportunity to suggest new hypotheses about techniques based on observations made on the monument itself, on comparisons and on a logic reasoning led by a double care of efficiency and economy.

Translation : Isabelle FAUDUT:T

Schlagwörter. Gallia Narbonnensis, Nîmes, Pont du Gard, Archilektur, Regulierbecken, Gerüstbau, Baustellenbetrieb, Hebevorrichtungen, Baumaterial und Bautechniken, Inschrift, antiker Wasserbau.

Zusammenfassung. Die jüngsten Forschungen zur Wasserleitung von Nîmes ermöglichten einen neuen Zugang zu Fragen der Konzeption des Pont du Gard und der bei seiner Errichtung angewandten Techniken. Sie betreffen die Anpassung an das natürliche Relief samt Platzwahl der Talquerung über den Gardon, die Konsequenzen aus dem variablen Gefälle der Kanalleitung, Siatik und Verformung des Bauwerks, die Versorgung der Baustelle mit Werksteinen, Kalk, Sand, Holz und Metall sowie Anzahl und Qualifikation der nötigen Arbeitskräfte.

Diese Neubetrachtung der Erbauung des Pont du Gard bietet auch die Gelegenheit, neue Hypothesen über die technischen Abläufe der Bauausführung zur Diskussion zu stellen, die sich auf Beobachtungen am Monument selbst, auf Vergleichungen, aber auch auf logische Überlegungen zu Effektivität und Ökonomie stützen.

Übersetzung : Stefan WIRTH

L'étude de la construction du Pont du Gard, ouvrage d'art principal de l'aqueduc de Nîmes, pose de nombreux problèmes spécifiques. Ils ont été plus ou moins abordés par nos prédécesseurs : A. Léger en 1875, E. Espérandieu en 1926, I. S. Nikolaev en 1967, C. O'Connor en 1993 ainsi que d'autres auteurs, en général peu rompus à l'étude des problèmes techniques.

Nous les examinerons successivement en essayant de les résoudre dans l'ordre normal où ils ont dû être traités par les constructeurs. Nous évoquerons d'abord ceux relatifs à la conception du monument, ceux qui concernent les matériaux et leur approvisionnement et enfin ceux qui se rapportent à sa construction proprement dite ${ }^{34}$.

\footnotetext{
34. Je dédie cet article à la mémoire de Léon Germand, conservateur du musée Requien en Avignon, qui, dans les années 1950, m’a appris à regarder la nature. L'essentiel de cet article a été rédigé au cours des premiers mois de 1998 pour servir de cadre à la réflexion préalable sur l'espace muséographique du Pont du Gard. Je tiens à remercier J.-L. Fiches, P. Gros, X. Lafon, M.-F. Giacobbi-Lequément et P. Varène qui unt bien voulu lire mon mantserit, en faire une critique constructive et me faire bénéficier de leurs conseils avisés.
} 


\section{LA CONCEPTION DE L'ARCHITECTE}

\section{LE CHOIX DU SITE}

I'architecte-ingénieur chargé du projet n'a pas conçu le Pont du Gard isolément. Une vision d'ensemble du tracé de l'aqueduc était indispensable et c'est dans ce contexte qu'il a intégré le problème du franchissement du Gardon. En revanche, l'inverse est aussi vrai. Le Pont du Gard étant l'ouvrage majeur de l'aqueduc de Nîmes, la possibilité de l'implanter à l'endroit du rétrécissement maximum de la vallée a constitué l'un des paramètres essentiels dont le concepteur de l'ouvrage a eu à tenir compte.

L'endroit choisi correspond en effet à un rétrécissement de la gorge (fig. 45). De part et d'autre de la vallée, des émergences de la roche calcaire du Crétacé laissaient espérer la possibilité d'asseoir correctement les fondations des piles du monument.

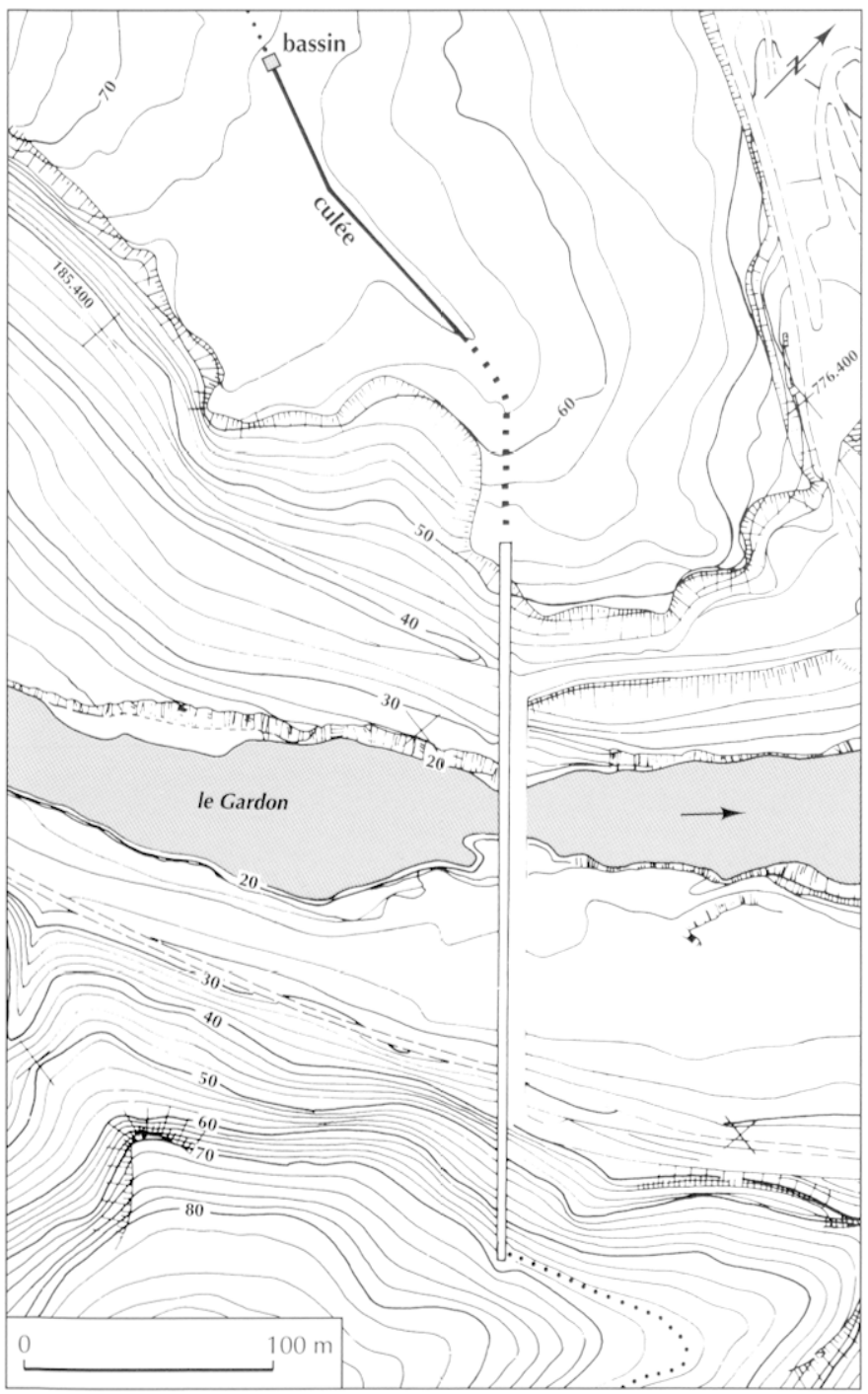

Fig. 45 - Plan topographique du Pont du Gard dans son environnement immédial aver la restitution des douze arches amont du niveau supérieur (dessin M. Sintès, CNRS).
En rive gauche du Gardon, le versant nord, relativement peu pentu, permettait un approvisionnement facile du chantier. Ce n'était pas le cas pour le versant méridional, en rive droite, beaucoup plus escarpé.

\section{L'ÉTABLISSEMENT DU PROJET}

Le concepteur a dû avoir en tête le souci permanent de construire à moindre coût un ouvrage solide et répondant aux exigences de sa fonction.

Le profil de la vallée, le Gardon lui-même et la position du fil d'eau dans la canalisation constituaient une autre série de paramètres majeurs du projet. Ils définissaient à eux seuls l'enveloppe de l'ouvrage à construire.

Nous en avons déjà parlé dans des publications antérieures (Fabre et al. dir., 1991, p. $72 ; 2000$, p. 99-101), mais il convient de rappeler ici que la hauteur du Pont du Gard a été déterminée par l'enterrement de la canalisation au franchissement du seuil de Clausonne à Sernhac. En effet, le librator ${ }^{35}$ est parti de ce seuil, en remontant vers l'amont et en affectant à l'aqueduc une pente minimale dans le but précis de réduire au maximum la hauteur du Pont du Gard et de tous les ouvrages d'art compris entre Vers-Pont-du-Gard et Remoulins. L'incidence de la hauteur du Pont du Gard sur celle du pont de la Sartanette n'était pas importante, mais elle était considérable sur les ponts à arcades continues de la Lône, du Pont-Rou et de Valive et sur les deux ponts à deux étages de Font Ménestière et de la Combe Roussière. Le choix de réduire la hauteur des ouvrages pour des raisons économiques évidentes a eu à son tour comme conséquence d'affecter à chaque tronçon du canal une pente différente ; forte entre Uzès et Vers-Pont-du-Gard, très faible entre Vers-Pont-du-Gard et le seuil de Clausonne, et à nouveau forte entre l'étang de Clausonne et Nîmes. Nous verrons par la suite que cette absence d'homogénéité a eu de graves conséquences sur le fonctionnement de l'aqueduc.

Le Gardon est une rivière capricieuse aux crues aussi fortes que subites, et tout ouvrage d'art dans son lit majeur se devait d'être porté par des piles suffisamment puissantes pour résister aux crues, mais aussi étroites et profilées de manière à réduire au maximum les poussées exercées par le flux liquide. Pour la même raison, il était nécessaire de prévoir un minimum de points porteurs et donc de franchir de larges portées par de grandes arches. Il fallait aussi élever le niveau de la naissance des arches à une hauteur suffisante pour que les eaux tumultueuses des plus fortes crues puissent passer sous les arches, sans risquer de provoquer par leur force un déversement de l'ouvrage. Il fallait donc construire un ouvrage composé de trois ponts superposés d'une solidité extrême et dont le

35. Nom antique du technicien qui effectuait les opérations de nivellement (Vitruve, De Archilectura, VIII, 5, 1-3 et commentaires p. 136-142; Pline le Jeune, Lettres, X, 41,3). Vitruve conseille l'emploi du chorobate, parce que plus précis, mais indique également la possibilité d'utiliser une libra aquaria (niveau d'eau fondé sur le principe des vases communicants) ou d'une dioptra, instrument polyvalent, longuement décrit par Héron d'Alexandrie, dont la fonction principale est de permettre la mesure des angles horizontaux et verticaux. la dioptra peut donc aussi servir à des nivellements (Ileromis Alexandrimi opera, 3, p. 193, Wiesbaden, Teubner Verlag). 
nombre de piles du premier niveau soit réduit au maximum. Ce double objectif nous parât être la raison essentielle pour laquelle le type de pont dit à piles entretoisées, aux arches de diamètre modeste et dont le nombre de points porteurs est important, a été écarté 36 .

\section{LE BASSIN RÉGULATEUR DE LA BALAUZIÈRE ET LA HAUTEUR DU PONT}

Comme la pente du canal n'était pas uniforme, il n'était pas possible à l'ingénieur antique de connaître a priori la hauteur de l'eau dans le canal. De fait, lors de la première mise en eau de l'aqueduc, le niveau de l'eau est monté plus haut que prévu dans le secteur le moins pentu. Dans la canalisation enterrée, ce n'était pas très grave, mais sur le Pont du Gard et les autres ouvrages aériens, d'éventuels débordements pouvaient les mettre en péril. Pour éviter de tels désagréments, un bassin de régulation muni de vannes a été construit en amont du Pont du Gard (Fiches, Paillet, 1989). Son implantation, sur le versant rocheux nord de la colline de la Balauzière et au tout début de la culée amont du Pont du Gard, est particulièrement judicieuse. En cas de tropplein, l'eau excédentaire était évacuée par l'intermédiaire d'un canal de décharge et ruisselait ensuite sur l'épiderme de la roche avant de rejoindre le Gardon en aval du pont (fig. 46).

Afin d'éviter des débordements lors de la première mise en eau, la vanne de décharge a dû rester ouverte d'un tiers. Pour ne pas perdre cette eau qui, à grands frais, avait déjà été transportée d'Uzès, le gabarit du canal fut augmenté de $0,60 \mathrm{~m}$ en hauteur. Pour résoudre ce problème technique, les dalles de couverture de la canalisation furent surhaussées de la hauteur d'une assise de blocs de grand appareil et le cuvelage de mortier de tuileau des parois verticales intérieures fut surélevé d'environ deux pieds (fig. 47).

$\mathrm{Si}$, sur le Pont du Gard, les dalles de couverture du canal ont été simplement replacées, il n'en a pas été de même pour tous les ouvrages d'art situés en aval. Ceux-ci ont été renforcés latéralement par des murs construits pour encaisser les poussées induites par la surélévation du canal. Ces murs étaient reliés entre eux par une arche mineure construite sous l'arche primitive et, à leur partie supérieure, par le massif de la nouvelle voûte qui couvrait le canal et les murs bajoyers épaissis.

\section{LA STATIQUE DE L'OUVRAGE}

Le concepteur du Pont du Gard n'en était probablement pas à son coup d'essai. Il avait certainement beaucoup voyagé, vu et construit d'autres ouvrages avant d'entreprendre le projet du franchissement du Gardon par l'aqueduc de Nîmes ${ }^{37}$ (fig. 48).

36. Le meilleur exemple de ces ourrages d'art dits à piles entretoisées est le pont-aqueduc dit de Los Milagros à Merida en Espagne (Grewe, 1985, p. 67). C'est aussi le cas du pont sur l'oued Bellah et des étages supéricurs du pont du Chabet Illelouine de l'aqueduc de Cherchell en Algérie (Leveau, Paillet, 1976).

37. In matin, par lumière frisante exceptionnelle, nous avons découvert, en 1989, un relief représentant un légionnaire srulpté sur le bossage de la clef de l'arc de tête oriental de la $6^{\circ}$ arche du $2^{\circ}$ niveau

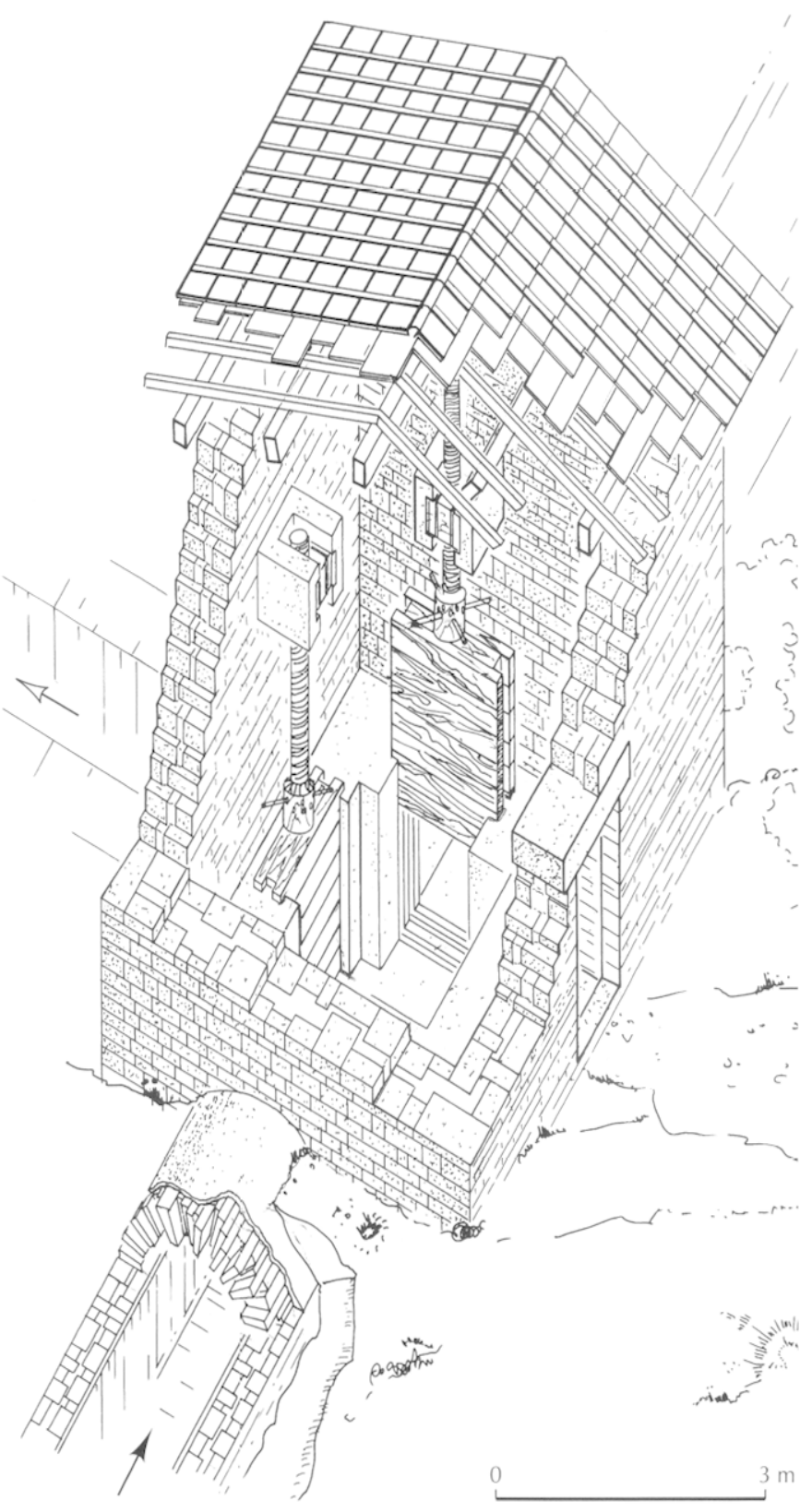

Fig. 46 - Proposition de restitution axonométrique du bassin de régulation du débit des eaux sur le Pont du Gard (dessin J.-L. Paillet et P. Neury, CNRS).

Le choix du point précis de l'implantation du pont, où affleurent des émergences de calcaire urgonien, était évidemment un facteur de stabilité de l'édifice. La définition de son profil en travers (coupe transversale) en était un autre. Ce dernier permet d'inscrire la section de l'ouvrage à l'intérieur d'un trapèze dont les bases inféricure et supérieure sont très différentes (5,92 $\mathrm{m}$ en bas et $2,97 \mathrm{~m}$ au sommet) et dont les

du pont. Nous avons alors supposé que le concepteur de l'ourrage était un légionnaire démobilisé ou affecté temporairement à cette charge civile (Fabre ef ol. dir., 1999; voir aussi une autre interprétation dans Bessac, 1999). 

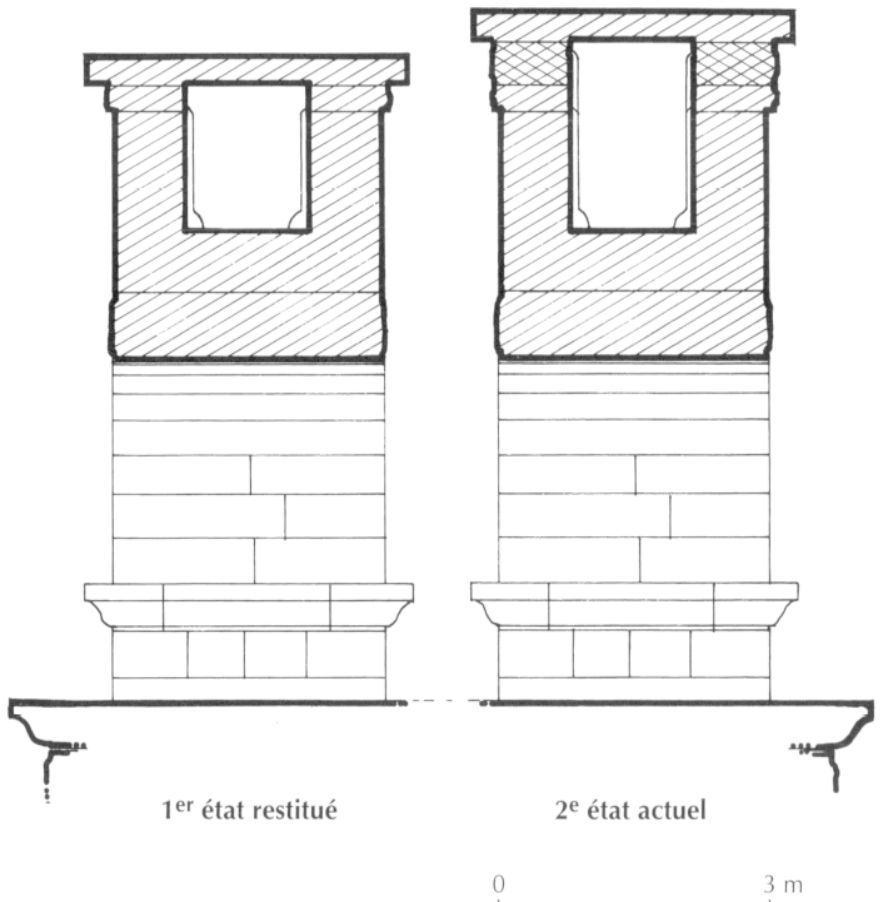

Fig. 47 - Profils en travers (coupe transversale), sur le Pont du Gard, de la canalisation dans son état primitif et après sa surélévation (dessin J.-L. Paillet, CNRS).

obliques présentent un fruit symétrique de $3,14 \%$. Ce profil est la conséquence de trois paramètres essentiels (fig. 49) : le premier relève de l'obligation de construire des piles larges pour pouvoir lutter efficacement contre la poussée des eaux du Gardon lors des grandes crues, le deuxième est une conséquence de la section relativement importante de la canalisation supérieure et le troisième tient, tout simplement, à la hauteur même de l'ouvrage.

Encore ne fallait-il pas trop réduire la surface au sol des points porteurs afin d'éviter que les pressions exercées par la masse du pont ne provoquent l'écrasement des blocs de grand appareil de sa base. Nous avons calculé que le volume de pierre mis en ouvre pour une travée courante du Pont du Gard est de $2438 \mathrm{~m}^{3}$ et que son poids est de $4632 \mathrm{t}$. La pression excrcée sur l'assise de fondation est de l'ordre de $16 \mathrm{~kg} / \mathrm{cm}^{2}$, soit le quart de la valeur limite de la résistance du matériau ${ }^{38}$. Il avait donc été prévu une importante marge de sécurité.

Malgré tout son talent, le concepteur n'a pas pensé à un autre problème. Nous ne saurions lui en tenir rigueur dans la mesure où la " pathologie " du monument consécutive à ce problème est relativement limitée et impossible à maîtriser. Le Pont du Gard, dans son état actuel, présente, sur sa rive droite, une déformation convexe du côté amont par rapport au Gardon. Cette déformation est importante à la hauteur de son troisième étage (environ $1,30 \mathrm{~m}$ ), faible à celle de son

38. La densité de la pierre du Pont du Gard est estimée à $1,9 \mathrm{t} / \mathrm{m}^{3}$ et la valeur limite moyenne de sa résistance à la compressionest de $70 \mathrm{~kg} / \mathrm{cm}^{2}$ (Noël, 1970, p. 123).

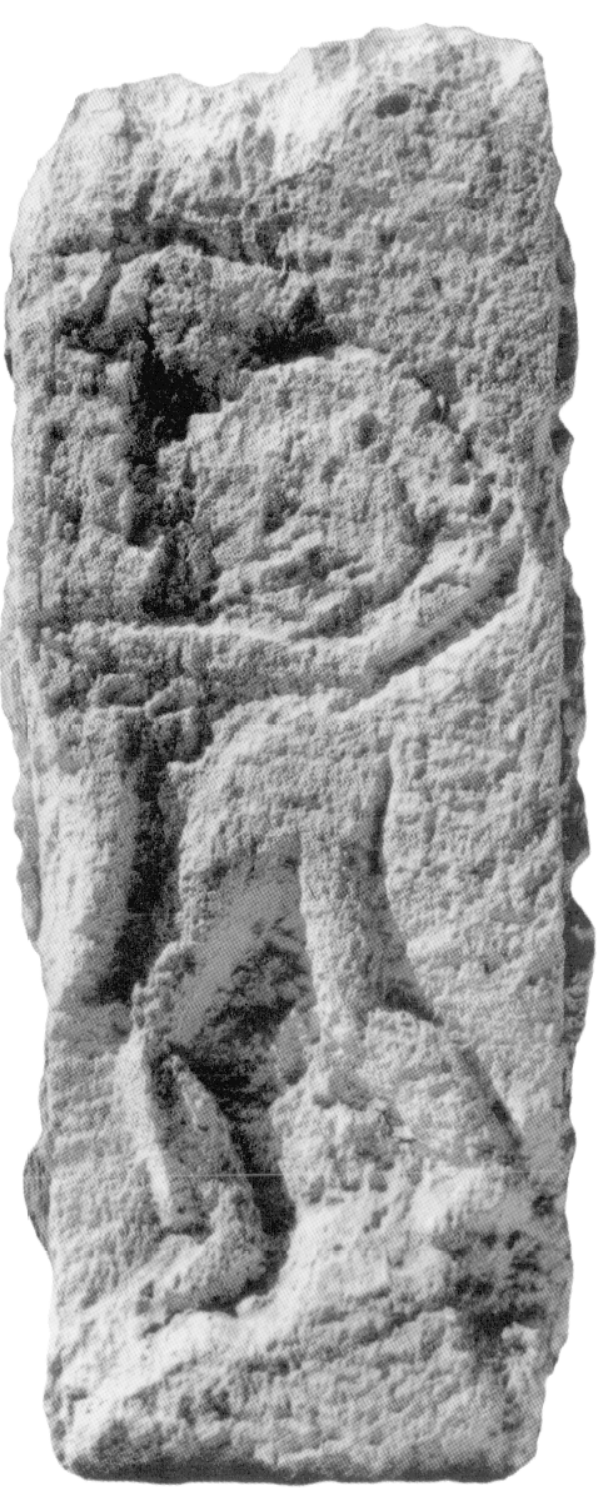

Fig. 48 - Moulage du relief sculpté sur le bossage de la clef de l'arc de tête oriental de l'arche majeure du $2^{2}$ niveau du Pont du Gard réalisé par J. Gonsalvez. Il s'agirait d'un légionnaire casqué passant à gauche et, apparemment, porteur d'un rouleau de plan. J.-C. Bessac y voit un carrier et M. Janon un gladiateur (cliché J.-L. Paillet, CNRS).

deuxième et nulle à celle de son premier niveau. De multiples hypothèses ont été émises sur l'origine de cette déformation. Certains ont pensé qu'elle était contemporaine de sa construction, soit volontaire afin de mieux lutter contre les crues du Gardon, soit involontaire, le mistral étant responsable de la déformation du cordeau du maçon ! D'autres ont pensé que les échancrures creusées au Moyen Âge dans les flancs ouest des piles, pour permettre le passage des charrettes, étaient à l'origine de cette déformation 39.

Reprenant le problème, nous avons successivement éliminé ces hypothèses ainsi que celle relative à un éventuel affaisse-

39. Voir la gravure de Poldo d'Albenas, 1560. Le rempiétement des piles a été réalisé sous l'égide des États du Languedoc en 1699-1704. 


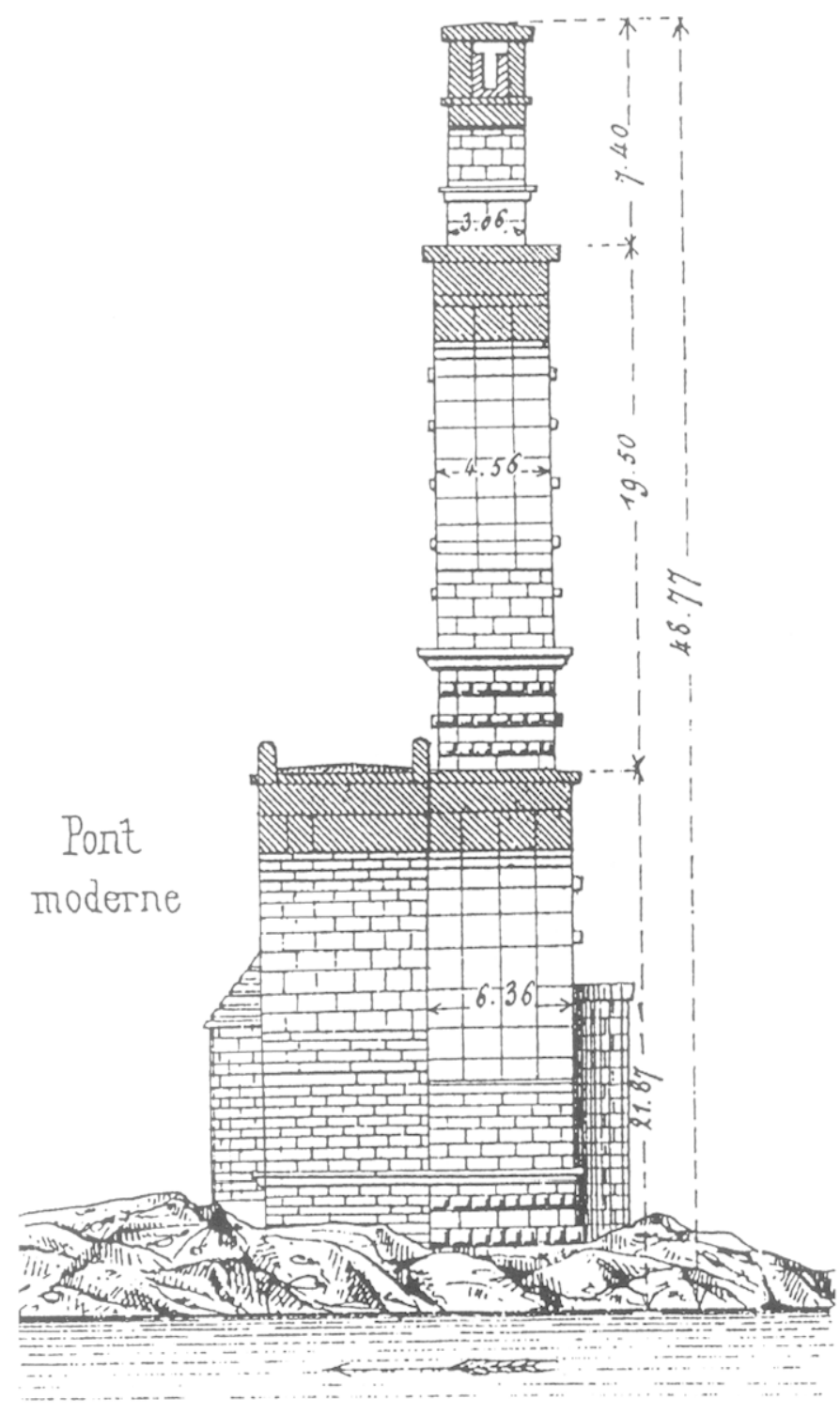

Fig. 49 - Profil en travers (coupe transversale) du Pont du Gard (d'après Léger; 1875, pl. L.X $X^{\text {tis }}$, fig. 2).

ment du sol qui, en cet endroit, est particulièrement stable. Des relevés topographiques très précis exécutés, en 1989, sous la direction de R. Martin, responsable du département de génie civil à l'IUT de Nîmes, ont abouti à la conclusion que le parement occidental du monument subit une dilatation diurne, variable en fonction de l'importance de l'ensoleillement. Cette dilatation a pour conséquence une déformation perpendiculaire à l'axe du pont de l'ordre de $5 \mathrm{~mm}$. La nuit, le monument reprend sensiblement sa place initiale avant de se déformer à nouveau le lendemain. En supposant que le monument, depuis sa construction présumée dans les années 50 apr. J.-C., ait subi cette dilatation 711020 fois (1 948 années $\mathrm{x} 365$ jours), on peut calculer la valeur résiduelle de sa déformation quotidienne permanente et cumulative soit $1,3 \mathrm{~m} / 711020=0,000018284 \mathrm{~m}$ par jour, soit environ 1,8 centième de millimètre par jour. À ce rythme, et en

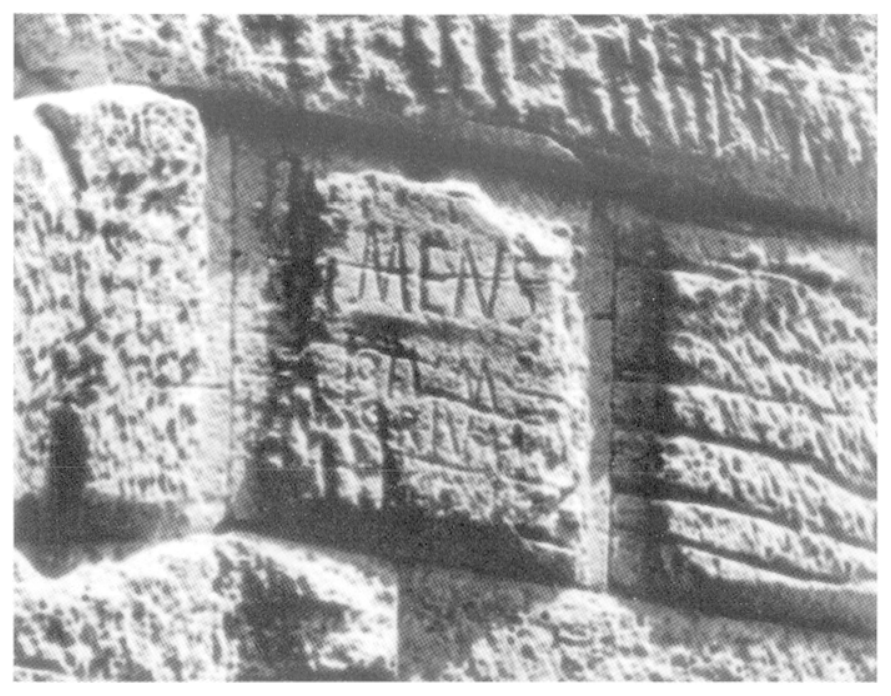

Fig. 50 - L'inscription MENS TOTIM CORIM redécouverte sur le Pont du (iard (cliché J.-L. Paillet, CNRS).

supposant que rien ne soit fait pour entraver ce phénomène, on peut estimer que l'ouvrage, dans 2492 ans, aura atteint une déformation égale à l'épaisseur même de son troisième étage et sera dans un état de déséquilibre très préoccupant!

\section{MÉTROLOGIE, TRACÉ RÉGULATEUR ET MAQUETTE}

L'unité de mesure qui a été utilisée pour la construction du Pont du Gard et l'ensemble de l'aqueduc de Nîmes est le pied antique classique de $0,2957 \mathrm{~m}$. La récurrence de cette mesure ou de l'un de ses multiples nous en a convaincu.

Dans la mesure où les constructeurs antiques ne disposaient ni d'appareils perfectionnés de topographie ni d'instruments de mesure très précis, il était indispensable que le conducteur des travaux pût, avec des méthodes simples, suive les indications marquées sur les plans dessinés par le concepteur de l'ouvrage. Le recours à des méthodes géométriques pour concevoir et dessiner de telles constructions, pour les reporter ensuite sur le terrain, est un héritage de la Grèce classique. Les architectes romains ont conservé et enrichi ces " recettes graphiques" et les ont répandues dans tout l'Empire. Une preuve manifeste nous en a été transmise par l'architecte concepteur du Pont du Gard qui a fait inscrire sur un bossage du parement occidental de l'un de ses avant-becs l'inscription : MENS TOTVM CORIVM (fig. 50) (Gascou, Paillet, 2000, p. 461-463). Le texte développé semble être : Mensum (est) totum corium et sa traduction littérale serait : "La totalité de l'épiderme a été mesurée ". En réalité, il semble que le message contenu dans cette courte phrase soit destiné au visiteur qui, regardant le monument, pouvait savoir que "la totalité de la composition de son épiderme, c'est-à-dire de ses façades, a été soigneusement mesurée et harmoniquement composée, suivant les règles de composition en usage à l'époque ". L'n tel message est si exceptionnel qu'il convient d'en souligner ici l'importance.

Sur le tracé régulateur du Pont du Card, plusieurs hypothèses ont également été formulées. Il semble que le diamètre 


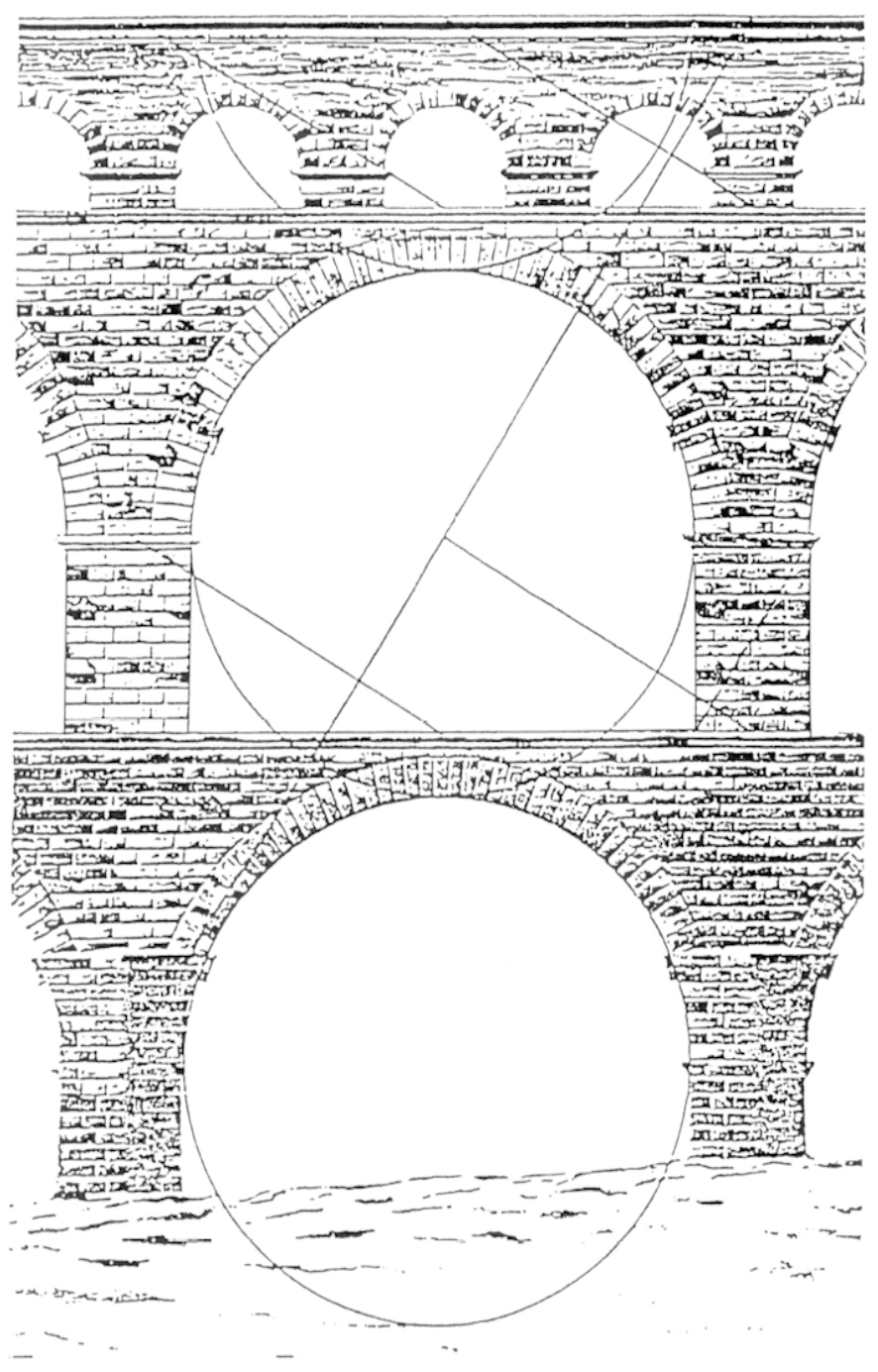

Fig. 51 - Hypothèse de proportionnalité de la section typique du Pont du Gard selon I. S. Nikolaev (1967a).

des arches du troisième étage du monument ait servi de paramètre principal. Le Pont du Gard a été conçu de telle manière que les piles des deux premiers niveaux soient superposées et qu'un nombre entier d'arches du troisième niveau s'inscrive dans le diamètre des arches des étages inférieurs. Ainsi, à l'exception de l'arche majeure, au-dessus du Gardon, sur laquelle quatre arches du troisième étage sont bâties, toutes les autres grandes arches n'en supportent que trois. Le même cintre a servi pour les arches supérieures car elles ont toutes le même diamètre. C'est la longueur des piles qui diminue et qui permet ainsi d'inscrire les quatre petites arches entre les axes des piles porteuses de l'arche majeure du Pont du Gard. La solution choisie donne l'illusion d'une parfaite régularité et procure l'impression d'une grande stabilité.

L'hypothèse de "proportionnalité de la section typique du Pont du Gard " de Nikolaev (1967a, p. 56-58) est intellectuellement intéressante, mais ne reste valable que si l'on accepte des approximations de l'ordre de $0,30 \mathrm{~m}$ lorsqu'elle est mise à l'épreuve sur un relevé précis ex dessiné à une échelle conve- nable (fig. 51). De plus, elle est nécessairement fausse car elle se fonde sur un relevé de l'état final du monument après sa restauration et sa surélévation antiques. Si tracé régulateur il y a eu, il ne peut avoir existé que sur un dessin de l'état projeté et donc originel. Sur ce sujet, nous resterons donc prudent.

Un tel monument n'a pas pu être construit sans avoir été, au préalable, dessiné en plan, en coupe et en élévation sur des papiers ou des parchemins. Nous n'en avons ici pas la moindre preuve archéologique ${ }^{40}$. Il est aussi probable qu'une ou plusieurs maquettes aient été réalisées.

\section{LES MATÉRIAUX \\ LE CHOIX DES CARRIÈRES}

La pierre locale, dite de Vers et de Castillon-du-Gard, est un calcaire molassique coquillier de couleur jaune clair et de bonne qualité ; il s'agit d'un matériau peu gélif qui résiste bien à la compression et qui est relativement facile à tailler (Anonyme, 1890; Noël, 1970, p. 118 et 122). La localisation des carrières est difficile à déterminer avec une absolue certitude car les fronts de taille antiques ont reculé au Moyen Âge et rares sont ceux où l'on peut découvrir des traces du pic de carrier pointu ou de l'escoude à deux dents utilisés pendant la période gallo-romaine. Il faut aussi avouer que les traces d'une escoude antique aux dents usées ressemblent à s'y méprendre à celles d'une escoude médiévale ou moderne à tranchant droit. L'argument le plus pertinent nous est offert par les dimensions des empreintes d'extraction des blocs de grand appareil. Ia taille des empreintes antiques est toujours très supéricure à celle des empreintes médiévales ou modernes.

La carrière la plus proche est celle dite de l'Estel située en rive gauche du Gardon et à quelque $500 \mathrm{~m}$ en aval du Pont du Gard. Récemment, les fouilles qui y ont été conduites ont abouti à la conclusion qu'elle était en service à l'époque antique ${ }^{41}$. Il y a tout lieu de penser que les blocs de pierre de taille qui en ont été extraits ont été utilisés pour la construction du Pont du Gard.

\section{L'EXTRACTION ET LE TRANSPORT DES BLOCS DE GRAND APPAREIL}

L'extraction dans les carrières antiques se pratiquait le plus souvent à ciel ouvert et à partir d'une rupture de pente qui permettait un front de taille facilement accessible. Après avoir

40. Des dessins gravés de l'élévation du temple d'Apollon à Didyme ont été découverts sur le parement intérieur des murs de sa cour par l.. Haselberger. De même, une épure, à l'échelle grandeur nature, du fronton du Panthéon de Rome a été identifiée sur l'esplanade dallée adjacente au tombeau d'Auguste à Rome (Haselberger, 1997). Voir également les Actes du colloque de Strusbourg où P. (Gros rappelle que nous ne disposons d'aucune preuve d'un dessin d'architecture prealable a la construction d'un bâtiment avant la Renaissance (Cros, 1985).

41. Après des sondages exécutés par.J.-(:. Bessac en 1992, de très importants dégagements et fouilles ont été réalisés en 2000 et 2001 sous la direction de M. Vacca-Goutoulli assistée par une équipe de l'INRAP' (Bessac, 1992 ; Bessac, Vacca-Goutoulli, 2002). 


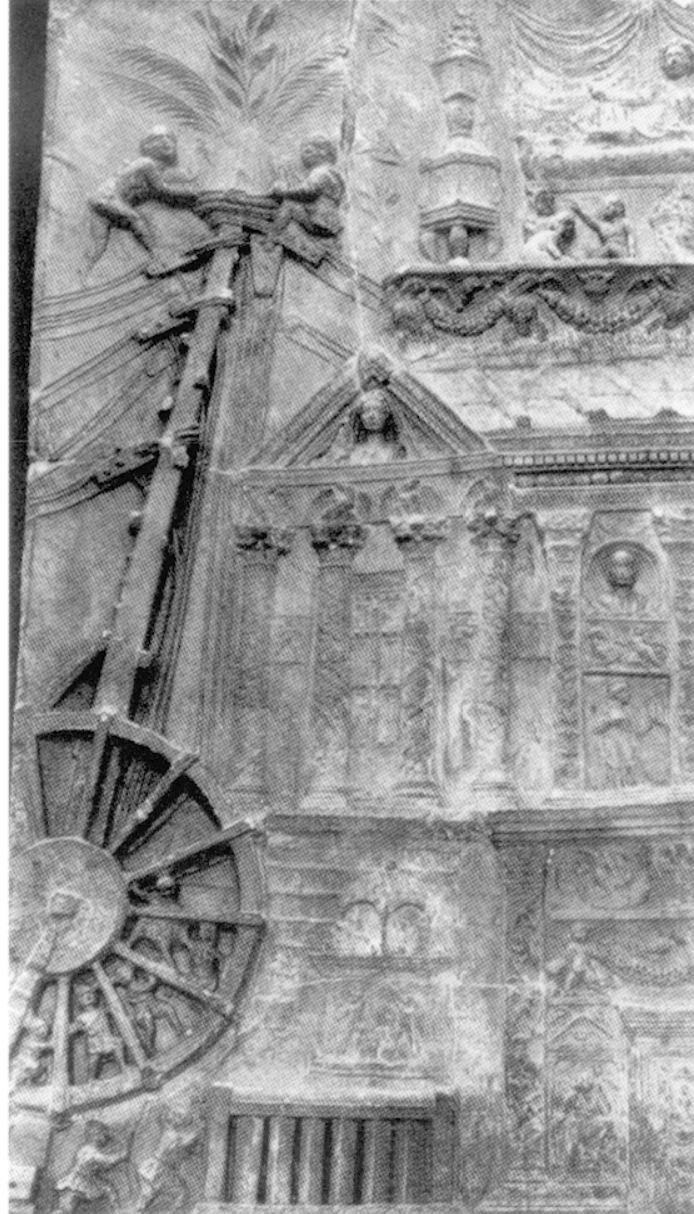

Fig. 52 - Bas-relief représentant le mausolée funéraire des Haterii en cours de construction (d'après Lugli, 1957, pl. XXX).

décapé la couche de terre arable et éliminé le premier lit de pierre altérée dit de découverte, les carriers attaquaient, à l'horizontale, le banc de pierre dont ils allaient tirer les blocs de grand appareil. Pour extraire les blocs, trois sillons verticaux, deux parallèles et un perpendiculaire, étaient creusés sur une profondeur égale et correspondant à l'épaisseur du bloc souhaité. Pour détacher le bloc de sa "semelle ", un sillon horizontal ou plusieurs cavités prismatiques étaient creusées à la base du bloc et suivant le lit de stratification naturel de la pierre. Dans ces " emboîtures ", des coins métalliques étaient insérés entre deux " paumelles". Des coups de masse répétés sur ces coins permettaient le décollement du bloc. Après vérification de sa qualité et de ses dimensions, il était roulé par « quartier » et, en fonction de sa destination, acheminé vers une aire de travail, puis vers un quai de chargement pour être posé sur un radeau ou une charrette tirée par des bœufs ou des mulets.

À en juger par l'importance des déchets de taille sur les sites des carrières, il est probable que les blocs bruts aient été épannelés et taillés avant leur transport. Cette pratique permettait aussi d'en réduire la masse et, par conséquent, de diminuer la dépense d'énergie pour leur transport.

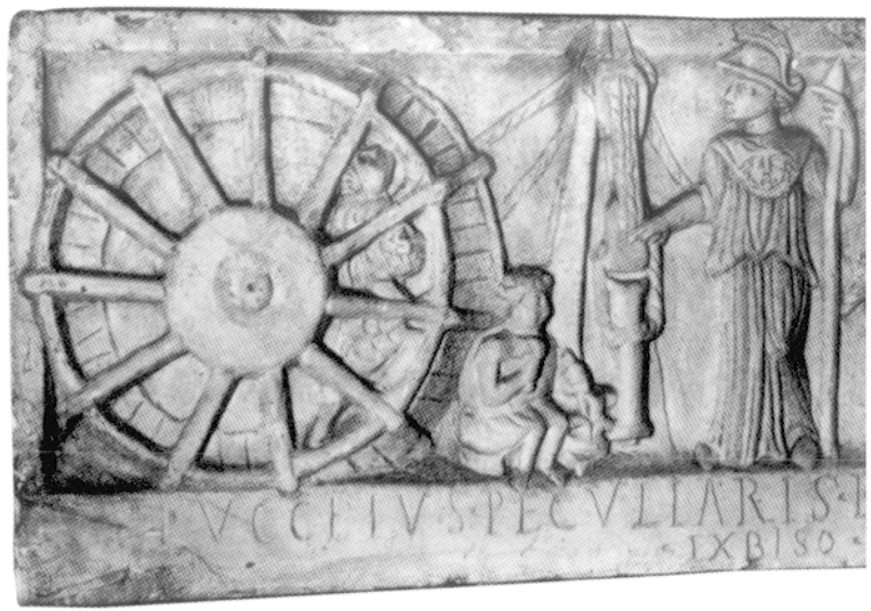

Fig. 53 - Bas-relief représentant la mise en ceuvre d'un fût de colonne découvert dans l'amphithéâtre de Capoue (d'après I.ugli, 1957, pl. XXX).

Pour déplacer les blocs, la pince métallique était utilisée. De même pour les soulever, la chèvre à deux pieds équipée d'un treuil à cage d'écureuil était couramment employée ${ }^{42}$ (fig. 52 à 54). Il est probable aussi que des mâts de charge ${ }^{43}$, semblables à ceux en usage dans la navigation à la voile, aient servi à la manutention et à la mise en œuvre des blocs de grand appareil. Grâce à ces méthodes, les carriers et les constructeurs romains ont pu soulever, déplacer et mettre en cuvre des blocs aux dimensions considérables. Ceux mis en ouvre au Pont du Gard sont loin d'être les plus volumineux connus, mais cela ne diminue pas pour autant l'importance du chantier qui, en Narbonnaise, reste certainement le plus considérable de l'époque.

\section{L'APPROVISIONNEMENT EN CHAUX}

La chaux était produite à partir du calcaire crétacique de l'Urgonien. Autour du Pont du Gard on le trouve sur les deux rives du Gardon, dans le secteur de la Balauzière et de la Croix de Martian en rive gauche et, en rive droite, dans les Bois de Remoulins. Nous n'avons pas retrouvé de four à chaux antique près du Pont du Gard. En revanche, au sud-est du village de Vers-Pont-du-Gard, nous avons repéré une vaste zone où le sol est jonché de moellons rubéfiés de calcarénite qui peuvent provenir de fours à chaux. Dans l'attente de fouilles nous resterons prudent parce que ce site est relativement éloigné des carrières d'Urgonien. Quoi qu'il en soit, l'approvisionnement

42. Nous ne citerons que les deux bas-reliefs les plus connus, celui des Haterii du musée du Latran et celui de l'amphithéâtre de Capoue, dont J.P. Adam a tiré d'excellents croquis de restitution (Adam, 1984, p. 48). Les traces archéologiques de ces engins de levage sont rares mais attestées, voir la restitution proposée par T. Kozelj in Sodini et al., 1980, p. 120 et fig. 83-84.

43. Des traces d'implantation de mâts de charge ont été reconnues en 1999 sur le site du trophée de Pompée au Perthus (Castellvi, communication personnelle) et, plus récemment, suite à la grande inondation du Gardon en décembre 2002 (Bessac, 2003). 


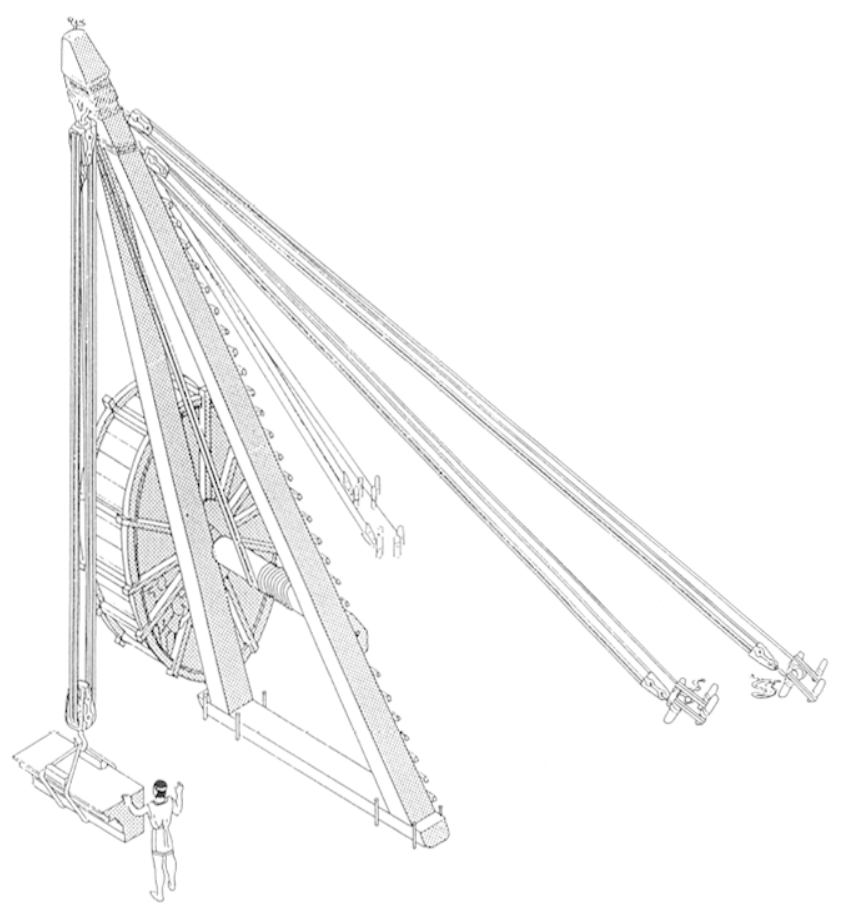

1

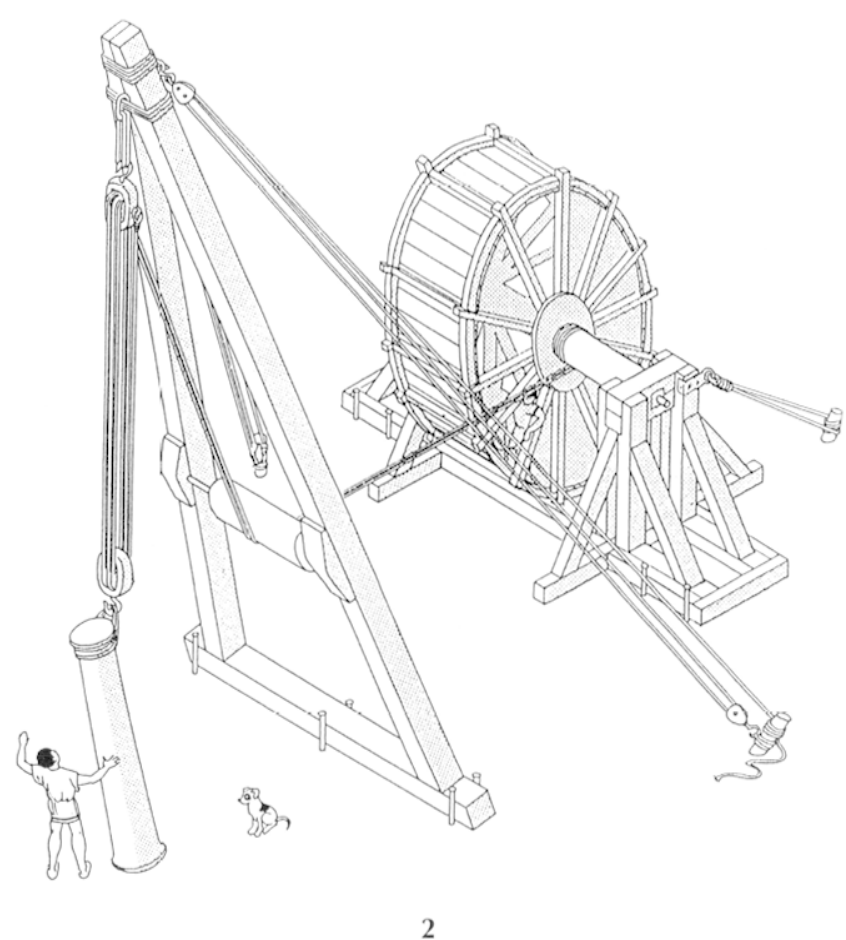

Fig. 54 - Propositions de restitution de la chère antique:

1, monument des Haterii (d'après Adam, 1984, p. 48, fig. 95); 2, bas-relief de Capoue (d'après Adam, 1984, p. 48, fig. 93 et 95). en chaux du chantier du Pont du Gard a pu se faire depuis les deux rives. Nous verrons ultérieurement que pour la construction du pont, on a eu recours à la chaux pour la maçonnerie de blocage des écoinçons compris entre l'extrados des grandes arches des deux étages inférieurs, les deux assises de leur couronnement et les parements latéraux en grand appareil (Laisné, 1859 : état de l'arcade $\mathbf{n}^{\circ}$ VII du premier rang). Dans les écoinçons du premier niveau du Pont du Gard, le volume de maçonnerie de blocage doit être réduit de celui, en grand appareil, qui constitue la base cachée des piles porteuses de la deuxième rangée des grandes arches de l'ouvrage. La hauteur de ce massif, essentiellement destiné à la transmission des charges verticales, est de l'ordre de $6,10 \mathrm{~m}$ : valeur comprise entre la naissance des arches courantes et le tablier du premier niveau du pont.

Le mortier de chaux a également été employé pour la construction des parements en petit appareil régulier (opus vittatum) des écoinçons situés entre les arches du troisième étage du pont, pour celle des parements des parois latérales du canal et pour la maçonneric de blocage coulée entre les parements des écoinçons et dans le libage des murs bajoyers du specus.

Un autre mortier de chaux mélangé à un cailloutis concassé et calibré d'Urgonien a servi à l'établissement du radier soigneusement nivelé. Enfin, les parements verticaux intérieurs du speecus ont été recouverts d'un enduit d'étanchéité constitué de chaux, de sable et de fragments de terre cuite pilés et calibrés. I.e solin bâti en même temps dans les angles inférieurs du canal a une constitution identique.

\section{LE SABLE}

Nous nous sommes aussi interrogé sur l'origine du sable contenu dans les mortiers du pont.

Au pied du monument, les rives du Gardon sont recouvertes par des alluvions sablonneuses plus ou moins riches

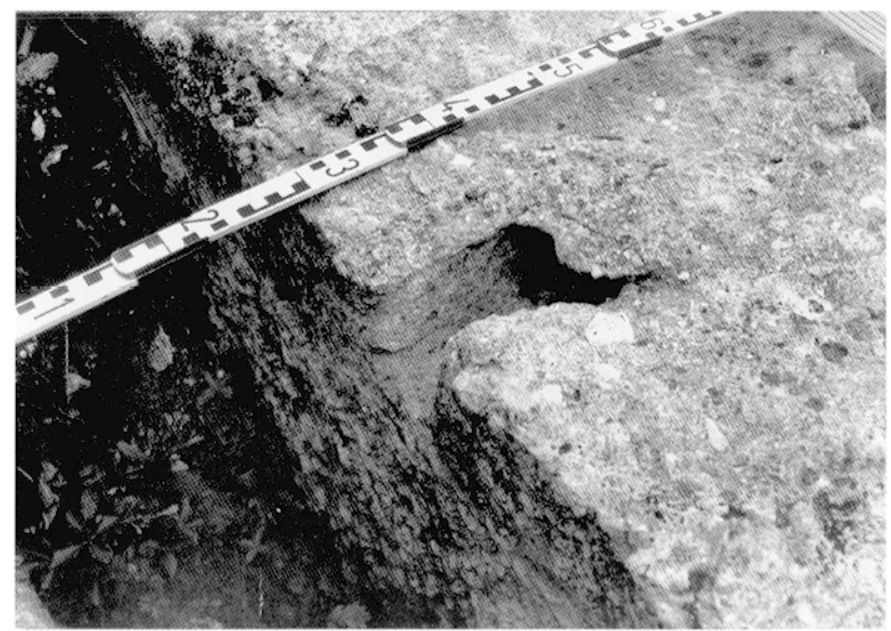

Fig. 55 - Trou de logement de crampon de bois reliant deux blocs de grand appareil de la culée amont du pont de Font Ménestière. Ia cavité était recouverte par un opercule de mortier de chaux qui portait, en négatif, les traces du sciage du crampon de bois (cliché J.-L. Paillet, (CNRS). 
en humus en fonction de la quantité de végétation qui les recouvre. Les sables lavés par les crues récentes sont évidemment les plus propres et les plus appropriés pour le gâchage des mortiers de chaux. Il convient toutefois de remarquer que le sable du Gardon n'est pas le meilleur des sables ; sa granulométrie est trop fine. De plus, il contient des parcelles de mica, de charbon et, en quantité infinitésimale, de l'or.

L'observation à la loupe binoculaire de fragments de mortier du libage des maçonneries du Pont-Rou et du pont de Valive prouve la présence de parcelles de mica et, plus rarement, de charbon. Cela est insuffisant pour affirmer que le sable utilisé pour la construction du Pont du Gard et des ourrages d'art adjacents provient du Gardon. Les facilités d'approvisionnement et d'exploitation de ces carrières à ciel ouvert et la quantité disponible constituent des arguments qui plaident en faveur de cette hypothèse ${ }^{44}$. Les analyses conduites sous la direction de $\mathrm{M}$. Schvoerer ont prouvé que le constituant du «bol rouge ", qu'Espérandieu supposait être du maltha, est un lait de chaux mélangé à un sable de quartz rouge d'une granulométrie précise et fortement chargé en oxyde ferrique (Schvoerer et al., 2000, p. 363). Les seules carrières de sable de quartz rouge actuellement connues sont à Bédoin, sur le flanc sud du mont Ventoux. Néanmoins, les prélèvements effectués prouvent que la dimension de ces grains de sable ne correspond pas à celle de celui utilisé pour la peinture intérieure ${ }^{45}$ du canal de l'aqueduc de Nîmes. G. Fabre nous a aimablement signalé la présence d'affleurements de sable de quartz rouge sur les communes de Saint-Quentin-la-Poterie et de SaintVictor-des-Oules qui ne sont qu'à une dizaine de kilomètres des sources de l'aqueduc. Nous les avons cherchés, mais pas encore trouvés.

\section{LE BOIS ET SON APPROVISIONNEMENT}

Nous verrons dans le chapitre suivant que, pour la construction de l'aqueduc de Nîmes et, en particulier, celle du Pont du Gard, trois types de bois étaient nécessaires : du bois de qualité moyenne pour les échafaudages, du bois de bonne qualité pour les ouvrages charpentés, cintres et chèvres, et du bois d'œuvre pour les machines, treuils, cabestans, moufles, poulies et crampons ligneux ${ }^{46}$ (fig. 55 ).

\footnotetext{
44. Pour les travaux de restauration du Pont du Gard, effectués entre 1855 et 1859 , J.-C. Laisné spécifie précisément l'origine des sables employés : "sables du Gardon".

45. La fonction la plus vraisemblable de ce badigeon semble d'avoir constitué un repère coloré sur l'épiderme intérieur du specus afin de signaler la présence de la paroi aux ourriers chargés d'éliminer les seules concrétions carbonatées.

46. Nous arons acquis la preuve que dans certains cas, les crampons étaient en bois, norés dans un mortier de chaux. C'était le cas lorsque le rôle affecté au crampon était d'assurer la cohésion pendant la seule durée du chantier ou celle du tassement naturel de l'œurre après sa construction. Sa fonction n'excédait alors pas sa durée de vie. Les blocs de grand appareil des culées amont du Pont-Rou et du pont de
}

Si les chênes de la région pouvaient servir à la fabrication des treuils, cabestans, moufles et poulies, il n'en était pas de même pour les bois de charpenterie dont les éléments étaient de grande longueur. Nous n'arons aucune certitude sur leur origine exacte, mais nous devons admettre que la solution la plus simple et la plus économique était de faire descendre les grumes des Cévennes par flottaison sur le Gardon. Les grands résineux des Cévennes pouvaient parfaitement convenir pour les cintres, les chèvres et pour les échafaudages.

\section{LE FER}

Sans fer, le Pont du Gard n'aurait jamais été construit. Il faut le dire et l'affirmer car le visiteur, émerveillé par ce colosse de pierre et ne voyant aucune trace d'un objet métallique, pourrait oublier ou, tout simplement, ne pas se douter que le Pont du Gard est un produit dérivé de l'invention et de la commercialisation du fer. Sans le fer, on n'aurait pas eu les outils suffisamment agressifs et solides pour extraire la pierre, tailler et scier les blocs de grand appareil, les lever à l'aide d'une louve, et les assujettir entre eux à l'aide de crampons ${ }^{47}$ (fig. 56).

Les techniques antiques d'extraction et de traitement du minerai de fer permettaient d'obtenir uniquement des outils en fer doux, suffisamment solides pour extraire et tailler la pierre. Toutefois, ils s'émoussaient rapidement et devaient être reforgés et trempés souvent ; d'où la présence probable de multiples forges autour du monument.

Les outils métalliques, relativement rares et précieux, se corrodent rapidement dans la terre ; cela explique que l'on n'en trouve guère dans les fouilles archéologiques. I.e seul outil que nous ayons découvert, en huit années de recherches sur l'ensemble des fouilles conduites sur l'aqueduc de Nîmes, est un ciseau de tailleur de pierre qui présente, à son extrémité postérieure, une douille conique prévue pour un emmanchement en bois (fig. 57). Cet outil a été recueilli dans le fond du vallon de la Combe Roussière. Isolé dans un tas de pierres et sans aucun contexte archéologique, nous ne pouvons pas affirmer qu'il soit antique. Il pourrait aussi dater de l'Antiquité tardive ou du haut Moyen Âge.

Font Ménestière étaient liés par des crampons de bois. Nous tenons à remercier J. Gonçalvez, qui nous a signalé qu'en arril 1998 deux crampons de bois ont été trouvés entre les blocs de grand appareil de la culée amont du Pont du Gard. D'après L. Chabal, qui en a effectué l'étude et la restauration, il s'agirait de bois de chêne. Ces crampons sont aujourd'hui conservés au Musée archéologique de Nîmes. Cette observation vient confirmer celle de J.-C. Laisné qui, en 1859 , signale la découverte de "queues d'aronde en chêne qui réunissaient les pierres entre elles ". Il précise, de plus, ne pas avoir trouvé de crampons métalliques.

47. Pour le Pont du Gard, nous supposons, en fonction de la qualité de l'œurre, que voussoirs, sommiers, boutisses saillantes, corniches, etc., étaient peut-être liées par des crampons métalliques de fer ou de bronze. Au pont de Bornègre, sur l'un des arrière-becs, une agrafe de fer scellée au plomb a été retrourée en place. J.-L. Fiches signale également l'utilisation d'agrafes en fer liant les blocs d'un avant-bec du pont d'Ambrussum (Fiches, 1970, p. 151). 


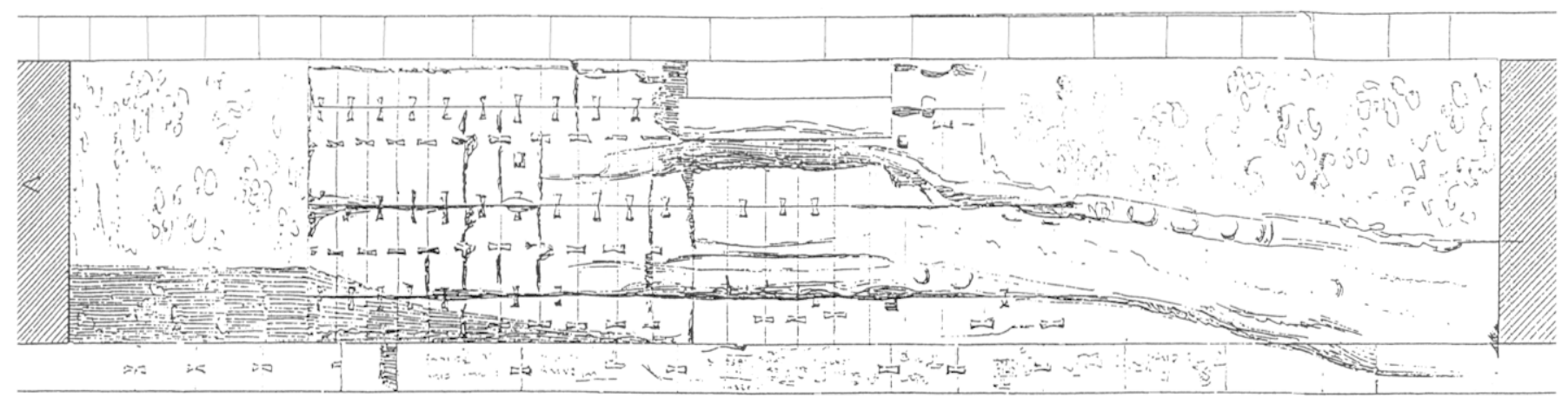

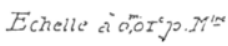

Fig. 56 - Plan de l'extrados de la voûte de la $5^{\circ}$ arche du premier niveau du Pont du Gard par l'architecte Laisné, chargé de la restauration du Pont du Gard. Ce dessin représente des cavités de crampons qui reliaient les voussoirs d'un même rouleau et les voussoirs des différents rouleaux (recherches de A. Pontet, archives des Monuments historiques, bibliothèque du Patrimoine, Paris).

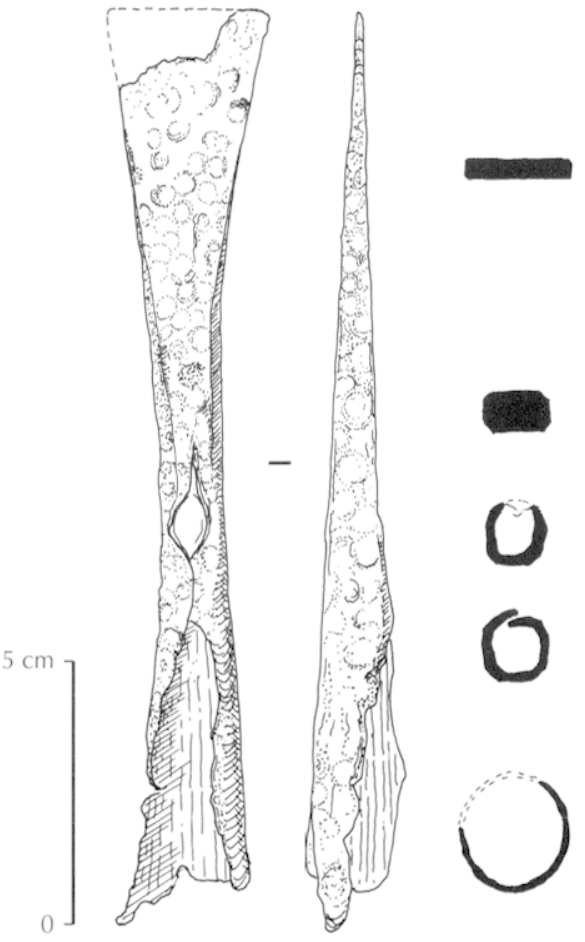

Fig. 57 - Ciseau à douille découvert au cours des fouilles en amont du pont de la Combe Roussiere (dessin J.-L. Paillet, CNRS).

\section{LA MISE EN CEUVRE ET L'ORGANISATION DU CHANTIER}

\section{LE CALIBRAGE DES BERGES DU GARDON}

Dans la mesure où il fallait construire dans la gorge un obstacle important, l'architecte antique a fait tailler la paroi rocheuse pour élargir le lit majeur du Gardon. Au droit de la grande arche qui enjambe la rivière, les berges, curieusement verticales, portent des traces de taille usées par le passage des eaux mais encore parfaitement visibles. Les constructeurs savaient que le Gardon avait des crues impétueuses et ont voulu élargir au maximum le goulot d'étranglement dans lequel l'essentiel des eaux passerait en de telles circonstances.

\section{LES ENTAILLES DES FONDATIONS DES PILES}

Pour asseoir les fondations des piles, des entailles horizontales ont été creusées dans le rocher et soigneusement aplanies afin de donner au lit de pose de la première assise de la fondation une assiette parfaite et ainsi éviter des pressions différentielles qui auraient pu créer des fissurations dans les blocs de grand appareil.

Le problème de la transmission des charges verticales étant capital, les blocs d'assise de fondation ont dû être rodés sur place à l'aide de "pinces à roder " ${ }^{48}$. Ce détail n'a été vérifié sur le Pont du Gard que récemment mais il l'a été, dès juillet 1982, pour un ouvrage mineur où nous en avons recueilli la preuve. Au droit de la partie détruite de la culée amont du Pont-Rou, les fouilles que nous avons conduites ont permis de mettre en évidence des trous parallèles creusés dans le rocher et situés devant des blocs en place qui possédaient, sur leur lit de pose, des cavités coudées adjacentes à l'une de leurs faces de joint. Les cavités coudées se situaient aussi toujours en face d'une des cavités verticales creusées dans le substrat rocheux. la relation entre les deux types de cavités était évidente et, à partir de ce constat, leur fonction le devenait aussi. Il ne pouvait s'agir que des traces d'un outil articulé ancré dans le substrat qui, grâce à un crochet coudé inséré dans la cavité creusée dans la face inférieure du bloc de grand appareil, permettait un rodage de son lit de pose sur le plan de l'entaille rupestre. Autant que notre enquête relative à l'origine, la chronologie et la diffusion géographique le permet, cette technique apparaît en Gaule en 72 av. J.-C. (trophée de Pompée à Panissars, commune du Perthus) et semble s'être diffusée dans les provinces occidentales de l'kmpire avant de disparaitre à la fin du III's. apr. J.-C:

48. Ia pince à roder est un outil que nous avons imaginé et dessiné en 1984, à partir de couples de cavités observées sur les vestiges du PontRou, en amont du Pont du Gard. Cet outil a aussi été qualifié de " pince à crochel " (Fincker, 1992). 


\section{LA MISE EN GEUVRE DES PILES ET DES ARCHES : LA PROGRESSION DU CHANTIER}

\section{LE PREMIER NIVEAU}

Au regard de la chronologie du chantier, une première évidence s'impose. Toutes les piles de l'étage inférieur du Pont du Gard ont pu être construites en même temps.

Pour la construction de celles situées en rive gauche, les blocs ont dû être apportés à pied d'œuvre sur des chariots tirés par des bœufs tandis que pour celles situées en rive droite, il a fallu poser les blocs sur des radeaux pour leur faire franchir le Gardon, les déposer ensuite sur des charrettes et les charrier jusqu'au pied des piles à édifier.

Les blocs déjà taillés recevaient au pied des piles les finitions indispensables juste avant que l'appareilleur n'ordonne leur levage et leur mise en place dans l'œuvre.

Pour la mise en place des blocs des assises inférieures des piles du premier niveau, des chèvres ou des mâts de charge d'une demi-douzaine de mètres de hauteur et posés sur le sol ont dû suffire. Au-delà, la chèvre ou le mât de charge devait être posé sur la pile elle-même, déplacé en fonction du bloc à positionner et surélevé à chaque assise. Ce travail de déplacement de l'engin de levage était délicat mais pas difficile. La tête de la chèvre, maintenue au milieu de la pile par des haubans accrochés à des amarres fichées dans le rocher, pouvait basculer à la demande vers le nord ou le sud. À chaque exhaussement, il fallait au préalable la placer verticalement et, à l'aide d'une paire de pinces, faire levier pour la soulever progressivement et alternativement sous chacun de ses pieds, de quelques centimètres à chaque fois pour ne pas la déséquilibrer. Dans le cas d'un mât de charge, on pouvait abattre le bras mobile pour diminuer la masse à exhausser, puis on soulevait le mât vertical de la même manière que la chèvre.

Pour ces opérations, des treuils étaient indispensables. Chaque treuil pouvait être soit solidaire de la chèvre soit indépendant. Pour que les appareilleurs puissent travailler correctement, il était souhaitable que les lits d'attente des assises déjà mises en place ne soient pas encombrés par la machinerie des treuils. Aussi, il est fort probable que chaque fois que cela était possible, les treuils étaient indépendants de la chèvre et installés en un endroit où ils ne gênaient pas. Ils étaient fort probablement amarrés dans le rocher au pied de chaque pile.

Les corniches qui servent d'impostes aux arches étaient construites de la même manière. I .es sommiers des arches et les premiers rangs des quatre rouleaux de voussoirs l'étaient également ${ }^{49}$ (fig. 58). Au-delà, il fallait poser les cintres.

49. Voir O'Connor, 1993, p. 172-173. Cet auteur indique que le modèle de voûte en berceau constituée de rouleaux séparés, mis en œuvre au Pont du Gard, n'est représenté statistiquement que dans $11 \%$ des arches des ponts antiques voûtées en grand appareil. Les autres modèles d'arches à joints croisés, à joints croisés et à double rang et enfin à joints croisés et à double rang alterné sont respectivement représentés dans $62 \%, 13 \%$, et $8 \%$ des cas. $\mathrm{C}$. O'Connor a répertorié quatre autres ponts antiques dont les arches sont construites en rouleaux ; il s'agit des ponts Saint-Martin ( 5 rouleaux) et de Chatillon près d'Aoste en Italie du Nord (dont ne subsiste qu'un rouleau), des ponts de Sommières dans le Gard (4 rouleaux) et de Boisseron dans l'Hérault (3 rouleaux).

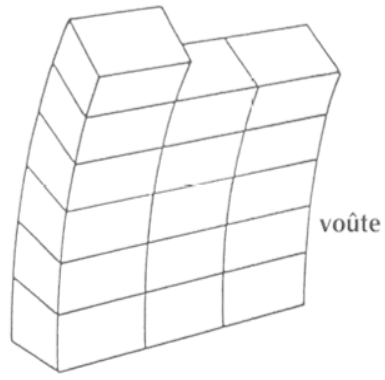

type A

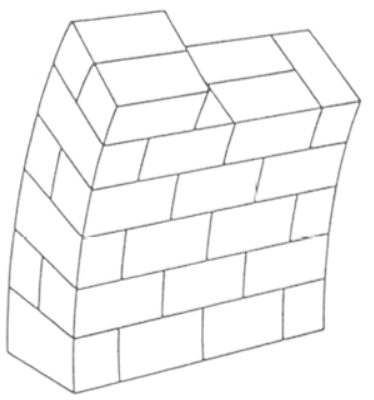

type C

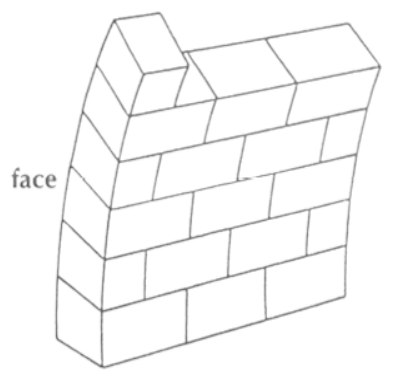

type B

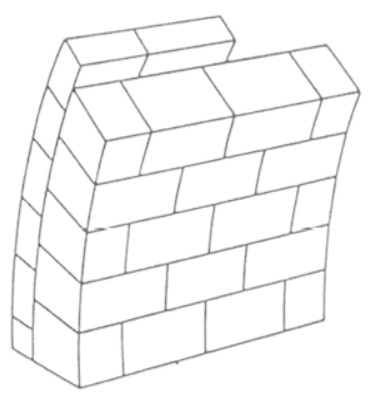

type D
Fig. 58 - Les différentes manières d'appareiller les voûtes des ponts antiques d'après O'Connor (1993, p. 172, fig. 142): type A, arche composée de trois rouleaux de claveaux séparés; type $B$, arche composée de claveaux posés en rupture de joint; type $C$, arche composée de claveaux posés alternativement en carreau et boutisse; type $D$, arche à deux rangées superposées de claveaux.

Tout d'abord, il convient de mettre un terme à une information erronée qui circule depuis fort longtemps à propos de la largeur des cintres qui ont servi à construire indépendamment les quatre rouleaux des arches. Il est impensable que le cintre n'ait eu qu'une largeur correspondant à un seul rouleau. Celui-ci aurait subi un effet de flambement sous l'effet de la moindre charge et l'ensemble se serait écroulé. Si c'était le cas, il aurait eu une largeur de $1,59 \mathrm{~m}$ pour une ouverture de $24,52 \mathrm{~m}$ pour l'arche majeure et de $19,20 \mathrm{~m}$ pour les autres grandes arches (fig. 59), ce qui n'est pas très réaliste. Les cintres, en conséquence, avaient toute la largeur des piles et si le choix constructif a été de créer des rouleaux séparés, ce n'est ni pour n'avoir à construire qu'un petit cintre, ni pour donner une très relative élasticité aux voûtes ${ }^{50}$ mais, au contraire, pour systématiser la taille des voussoirs qui en fonction de ce choix étaient tous les mêmes. Le travail des tailleurs de pierre en était considérablement simplifié. Si les voussoirs avaient été disposés en rupture de joint, il eût été nécessaire de les tailler les uns après les autres afin de les ajuster l'un à l'autre au fur et à mesure de leur mise en ouvre. Nous verrons par la suite que dans le cas du Pont du Gard cette préfabrication en série a été

50. Laisné indique dans son rapport daté de 1859 que tous les voussoirs étaient liés entre eux par des crampons (voir fig. 58). Cela exclut définitivement que l'un des rouleaux ait pu avoir un mourcment indépendamment des autres. 


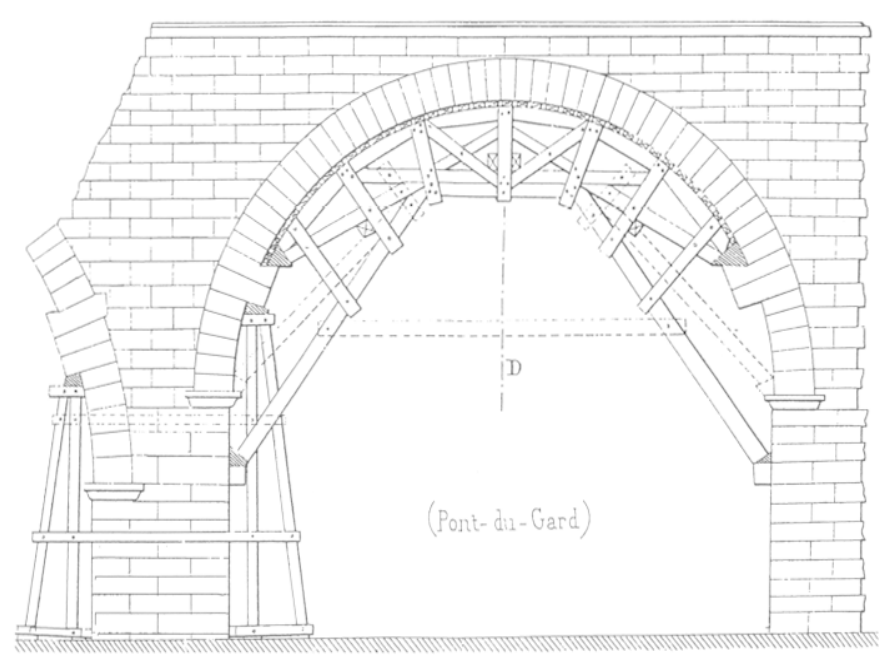

Fig. 59 - Morphologie du cintre d'une arche courante du $2^{\prime}$ niveau du Pont du Gard (d'après I.éger; 1875, fig. 13).

un facteur primordial du choix du parti constructif. Ce choix, évidemment, est aussi économique. Le coût de la construction du Pont du Gard était important et le concepteur a essayé de trouver une solution technique économique, facile à mettre en cuvre mais qui ne soit pas en contradiction avec son souci de l'esthétique et de la stabilité de son ceuvre.

Du point de rue de la chronologie de la construction des arches, il semblerait judicieux que la première arche construite ait été celle située en rive gauche du Gardon. La deuxième, qui enjambe la rivière, fut mise en ouvre immédiatement après et ainsi de suite jusqu'à la dernière arche du premier étage du pont. Si nous proposons, pour la construction de ces arches, une progression d'ouest en est, c'est parce qu'en procédant ainsi on pouvait facilement charrier les blocs au niveau même de leur mise en ouvre sans avoir à les lever sur 15 à $20 \mathrm{~m}$ de haut. Les chariots pouvaient facilement emprunter le tablier du pont nouvellement édifié avant que leur déplacement ne soit rendu impossible par la construction des piles de l'étage intermédiaire de l'ouvrage. Certes, on pourrait opposer à cette hypothèse que les animaux de trait risquaient en manœuvrant de provoquer des accidents très graves, aussi proposons-nous un charroi terminal, sur le tablier horizontal du pont, tracté par des hommes.

Avant d'appareiller les voussoirs de pierre, il fallait mettre en place un grand et unique cintre sous chaque arche. Probablement, le concepteur aurait souhaité, pour des raisons économiques, que toutes les arches soient semblables afin de pouvoir réutiliser le même cintre. La topographie du site l'en a empêché. Le franchissement de la rivière imposait une grande arche et, par conséquent, un cintre utilisable une seule fois dans le premier niveau; deux autres types de cintre ont permis la construction des sept autres arches. Nous devons en conclure que le concepteur a réellement pensé l'économie générale du projet et que les arches n'ont pas toutes été construites en même temps, mais au contraire l'une après l'autre suivant un ordre judicieusement programmé et raisonné.

Pour le détail des cintres nous acceptons le profil proposé par A. Léger, repris par E. Espérandieu et plus récemment par
J.-P. Adam, à la nuance près que nous élargissons ces cintres à la largeur totale de la pile (Léger, 1875, fig. 13 ; Espérandieu, 1926, p. 31 ; Adam, 1984, p. 190-191). Ces cintres étaient préparés et pré-assemblés au sol, puis démontés. Chaque pièce du cintre était ensuite levée puis mise en place et chevillée à partir d'un échafaudage provisoire. Pour soulever les pièces de bois des cintres, un engin de levage était indispensable. Deux chèvres, une sur chaque pile, pouvaient les soulever sans difficulté $^{51}$ (fig. 60). Le calage des appuis des cintres sur les voussoirs débordants et les contrefiches assises sur des bacs à sable sur les corniches débordantes permettaient, assurément, de les assujettir à leur emplacement.

En revanche, la mise en place des voussoirs des quatre rouleaux posait un autre problème. La chèvre que nous supposons sur la pile ne pouvait manifestement pas mettre en place les voussoirs de la partie médiane des arches. Le ballant de la chèvre était insuffisant pour porter $3 \mathrm{t}$ en bout de flèche à $12 \mathrm{~m}$. Si cela avait été le cas, il aurait fallu utiliser une chèvre de plus de $20 \mathrm{~m}$ de haut, et son amarrage sur le sommet de la pile eût été impossible. Une grue tournante avec une flèche d'une douzaine de mètres serait encore plus efficace que la chèrre, mais son empattement au sol aurait couvert la totalité de la pile. De plus, le problème de la longueur de la flèche tournante reste identique au cas précédent et non résolu. I.es premiers voussoirs de la partie médiane des arches ont pu être mis en place progressivement au fur et à mesure que l'écoinçon compris entre deux arches était surélevé (fig. 61, n 1). La chève ou le mât de charge pouvait ainsi s'avancer et appareiller en débordant de l'aplomb des piles. Mais pour les quelque quarante voussoirs du centre, cette solution peut difficilement être envisagée car elle aurait pu déstabiliser l'ouvrage, sauf si l'on prenait la précaution de faire lever, en même temps, par deux chèrres ou mâts de charge, deux claveaux symétriques par rapport à un axe vertical de l'arche. Cette solution est possible mais pour le moins délicate.

Aussi nous avons été conduit à rechercher une autre solution. En partant du postulat que toute technique de construction doit avoir laissé ses propres stigmates sur le monument, nous l'avons analysé de nouveau en fonction de cette recherche spécifique. Ce faisant nous avons constaté que la partie basse du premier niveau du Pont du Gard ne comporte pas de boutisse saillante sur ses faces latérales. Ces boutisses n'apparaissent qu'au-dessus de la naissance des arches, dans les écoinçons. Cette observation corroborée par celle relative aux boutisses saillantes de l'étage intermédiaire nous a permis d'imaginer que, si la partie basse du premier niveau du pont a été construite à l'aide de chèvres, la mise en ouvre des arches a contraint les constructeurs à monter un pont roulant supporté latéralement par deux poutres en treillis accrochées aux boutisses saillantes sur les faces latérales du monument

51. Nous avions pense, dans un premier temps, que les cintres araient été construits au sol, puis levés et mis en place déja assemblés. liexpérience de la restitution en grandeur réelle d'un petit cintre en châtaigner d'une arche du $3^{\circ}$ étage du pont dans l'espace muséographique du Pont du (ard nous a conduit à modifier notre hypothèse. Son poids, de l'ordre de $1500 \mathrm{~kg}$, suppose que pour les grandes arches. au moins, les cintres étaicnt lacós en pièces détachées, puis assemblés dans l'espace. 

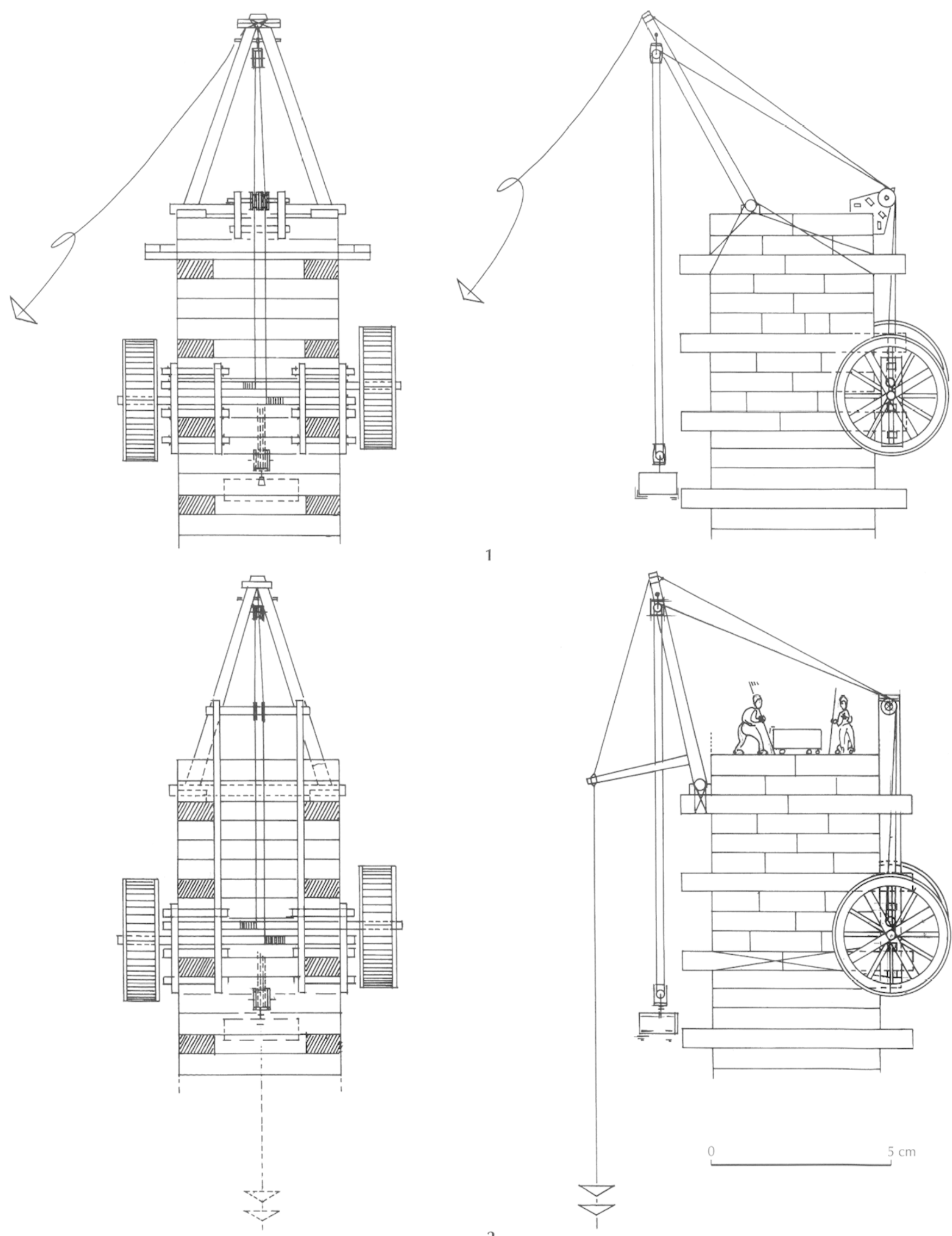

1



2

Fig. 60 - Deux solutions de positionnement de chères de petites dimensions pour le levage et la mise en place des blors des piles et des rintres du $2^{2}$ niveau du Pont du Gard: 1, la chèrre est sur la pile; 2, la chère est posée sur deux boutisses saillantes (dessin J.-L. Paillet, CNRS). 


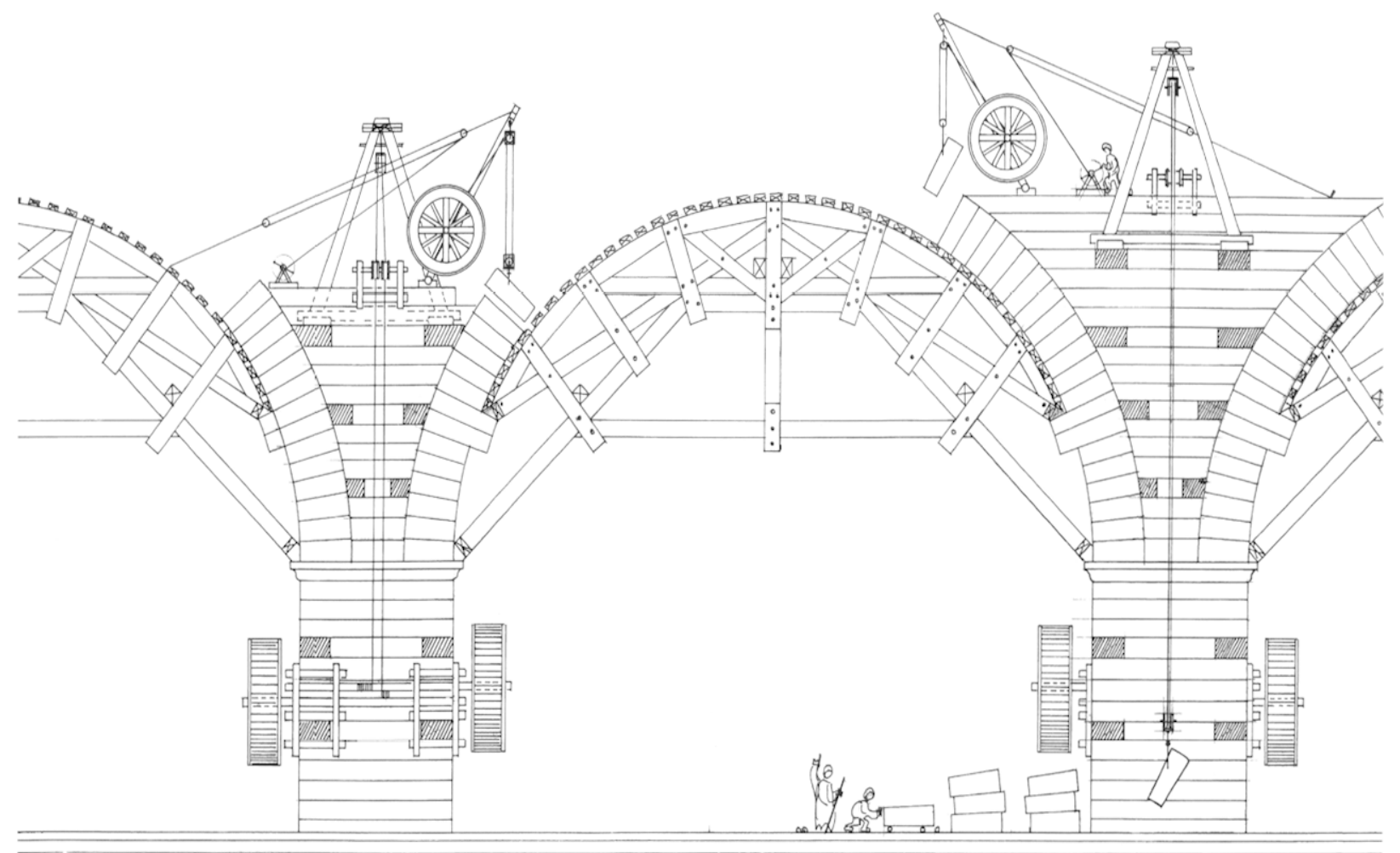

1

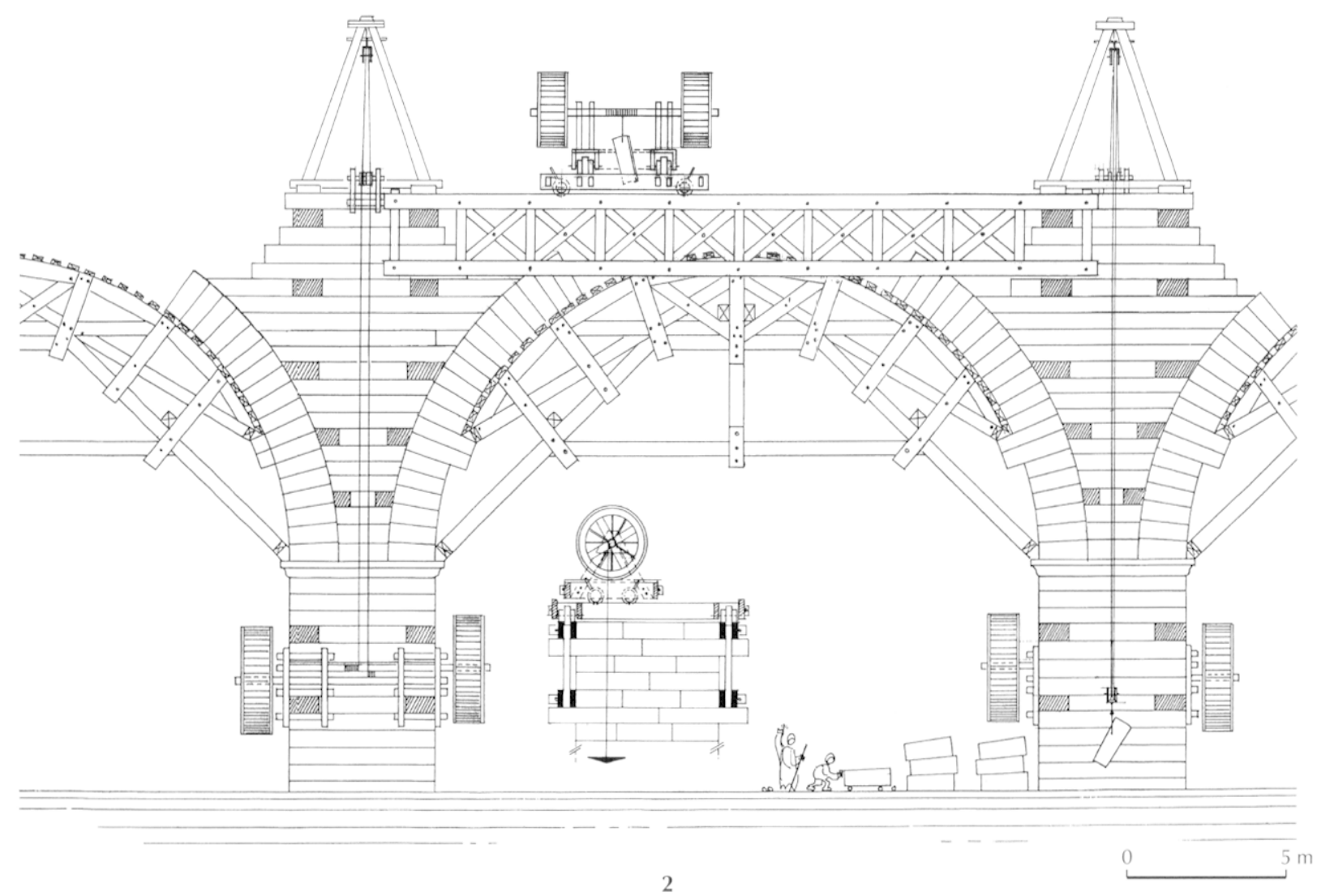

Fig. 61 - Arches du 2' niveau du Pont du Gard : 1, appareillage des premiers voussoirs, la chìre à treuil et tambour indépendlants posée sur les boutisses saillantes servait au levage des blocs et des voussoirs; la seconde, au treuil solidaire de la chòre, était utilisép ponor l'appareillage des voussoirs; 2, appareillage des derniers voussoirs, les chères sur les écoingons servaient ì hisser les blocs depuis le tablier du premier niveau. I.es treuils assujettis aux boutisses saillantes étaient à double effet; un tympanum pour lever les blors et l'autre pour modifier l'inclinaison de la chère. Le dessin sous l'arche correspond à une vue transversale sur les poutres treillis et sur le pont roulant (dessin J.-L. Paillet, (CNRS). 
(fig. $61, \mathrm{n}^{\circ} 2$ ). Un réexamen de la position précise des boutisses, en restituant celles dont les traces subsistent sous forme de fracture, confirme la validité de cette hypothèse. Certes, la poutre treillis est une locution qui, dans notre oreille, a une connotation très contemporaine mais, si on la remplace par " poutre composée " ou " poutre triangulée ", notre réticence s'estompe plus facilement. L'iconographie nous informe qu'à l'évidence la technique de la poutre treillis était connue. Avant nous, C. O'Connor, dans sa remarquable synthèse sur les ponts romains, indique que les ingénieurs antiques possédaient une connaissance empirique de la poutre treillis (O'Connor, 1993, p. 132-144 et fig. 112-118). Les représentations de poutres treillis sur les ponts et les ouvrages charpentés sur la colonne Trajane (fig. $62, \mathrm{n}^{\mathrm{os}} \mathrm{l}$ et 2 ) et sur celle de Marc Aurèle (fig. $62, \mathrm{n}^{\mathrm{N}} 3$ et 4 ) en sont les meilleurs témoignages (Monti, 1980, p. 45, 48, 66, 70-6, 70-7, 73-3, 73-8 et p. de couverture).

Bien sûr, la colonne Trajane est plus récente d'un demisiècle que le Pont du Gard, mais le pont de Narni sur la Nera ${ }^{52}$, en Ombrie méridionale, dont l'arche majeure mesurait plus de $32,10 \mathrm{~m}$ de diamètre (43 $\mathrm{m}$ selon les auteurs anciens), est augustéen ${ }^{53}$. Cette référence est d'autant plus intéressante que le pont de Narni possède sur ses faces latérales des boutisses saillantes semblables à celles du Pont du Gard. Dans cette optique, le pont de Narni serait l'archétype technique du Pont du Gard.

La hauteur des poutres treillis correspondait probablement à celle comprise entre deux boutisses saillantes superposées, soit 2,50 m environ. Elles devaient supporter un pont roulant mobile dans les deux sens sur des roues à gorge, dont le déplacement longitudinal était actionné à distance par des treuils ancrés au sommet des piles et amarrés aux boutisses saillantes des parements latéraux des piles. Le déplacement transversal était aussi possible à partir de la manœuvre d'un treuil à main ou d'un cabestan installé sur le plateau mobile supérieur du pont roulant. Enfin, un treuil, installé au milieu du plateau supérieur du pont roulant, permettait, grâce à deux petits tambours (cages d'écureuil) et à un moufle à quatre ou six brins, de soulever les charges les plus lourdes.

À l'aide de ce pont roulant, il devenait très facile de lever et appareiller les voussoirs des quatre rouleaux de la partie centrale des arches.

Avant de passer à l'étude de la construction du deuxième niveau du Pont du Gard, il convient de s'attarder sur trois problèmes fort importants qui sont valables pour la totalité de la construction en grand appareil de l'ouvrage.

Il s'agit, pour le premier, de la transmission parfaite des charges verticales, déjà évoquée à propos du couple de cavités identifiées au Pont-Rou dans le substrat rocheux et le lit de pose de la première assise de fondation de sa culée amont. Quand il

52. Voir Lugli, 1957, vol. II, t. LXXXII-1 ; Gar\%ola, 1963, n 60 ; Coarelli dir., 1980, p. $50 ; 0^{\circ}$ Connor, 1993, p. 78-80.

53. L'arche du pont Saint-Martin, sur le Lus, qui mesure $35,60 \mathrm{~m}$, date probablement des années 25 ar. J.-C.. mais sa construction n’a pas posé les mêmes problèmes que dans les cas de Narni et du Pont du Gard (O'Connor, 1993, p. 89-90). Quelques auteurs le vicillissent d'un siècle, 141-120 av. J.-C. s'agit d'une pile d'aqueduc, il arrive nécessairement un moment où l'on doit mettre en place le dernier bloc d'une assise. La présence des autres blocs déjà mis en place et rodés grâce à la pince à crochet articulé interdisait, pour ce dernier bloc, qu'il soit à son tour rodé. Comme cela n'était pas admissible, en fonction du parti pris volontaire de transmission parfaite des charges verticales, une solution étonnante et tout à fait astucieuse a été mise en œuvre. Un très gros bossage a été préservé sur le parement du bloc de l'assise qui allait recevoir le dernier bloc de l'assise suivante, de telle manière que la partie verticale de la pince à roder puisse trouver un point d'ancrage dans une cavité creusée à cet effet (fig. 63). Pendant le rodage du bloc, le préposé à la mancuvre ne pouvait être que sur le lit d'attente du bloc et, par conséquent, bougeait en même temps que le bloc qu'il était en train de roder. Pour cette manœuvre délicate, l'ouvrier devait être attaché et maintenu par l'un de ses collègues afin d'éviter la chute dans le cas d'un geste maladroit. Après le rodage, le bossage du bloc inférieur qui avait servi de point d'ancrage était abattu et sa face vue était ensuite parementée avec le même soin que tous les autres blocs. Heureusement, sur l'ensemble du monument, un seul bloc a été moins bien traité et c'est grâce à lui que nous avons pu comprendre et reconstituer la technique et son mode opératoire (fig. 64).

Le deuxième problème, proche du précédent, concerne le rodage au sol des faces de joint des voussoirs dont la planéité devait être parfaite. Deux observations concomitantes nous conduisent à affirmer que ces faces de joint faisaient l'objet d'un polissage par rodage d'un bloc sur l'autre. Pour ce polissage un abrasif particulier de couleur rouge était utilisé, car il a laissé sa teinte ocre rouge sur des faces de joint que nous avons pu observer, en 1997, aussi bien sur les voûtes du " temple de Diane " à Nîmes que sur le Pont du Gard au cours des travaux de restauration. La couleur rouge est produite par un sable de quartz contenant une forte quantité d'oxyde ferrique que nous supposons provenir des carrières d'ocre, proches de Saint-Quentin-la-Poterie et de Saint-Victordes-Oules. L'utilisation de ce sable abrasif pour roder et surfacer les faces de joint des voussoirs augmentait l'efficacité du frottement et permettait une économie de temps. Ce détail est important parce qu'il explique la raison pour laquelle les claveaux ont été marqués sur leur douelle. Théoriquement, dans une arche, tous les voussoirs d'un même rouleau sont semblables et ils n'ont, en conséquence, aucune raison d'être marqués. Si chaque face de joint est surfacée, rodée et polie avec celle qui va s'appuyer sur elle, le marquage devient indispensable.

Le troisième problème est relatif au sciage des joints. Nous savons que les joints verticaux étaient sciés de manière à ce que les imperfections de la taille disparaissent et que la forme des parements de deux blocs contigus s'épouse parfaitement. Cette opération est consécutive à la mise en place d'un bloc contre un autre et, pour exécuter ce sciage, l'appareilleur, sciotte ${ }^{5 t} \mathrm{en}$ main, pouvait soit se pencher dans le vide pour exécuter son œurre, soit se placer devant le joint, c'est-à-dire hors de la pile et nécessairement sur un échafaudage.

54. Petite scie utilisée pour scier les joints. 

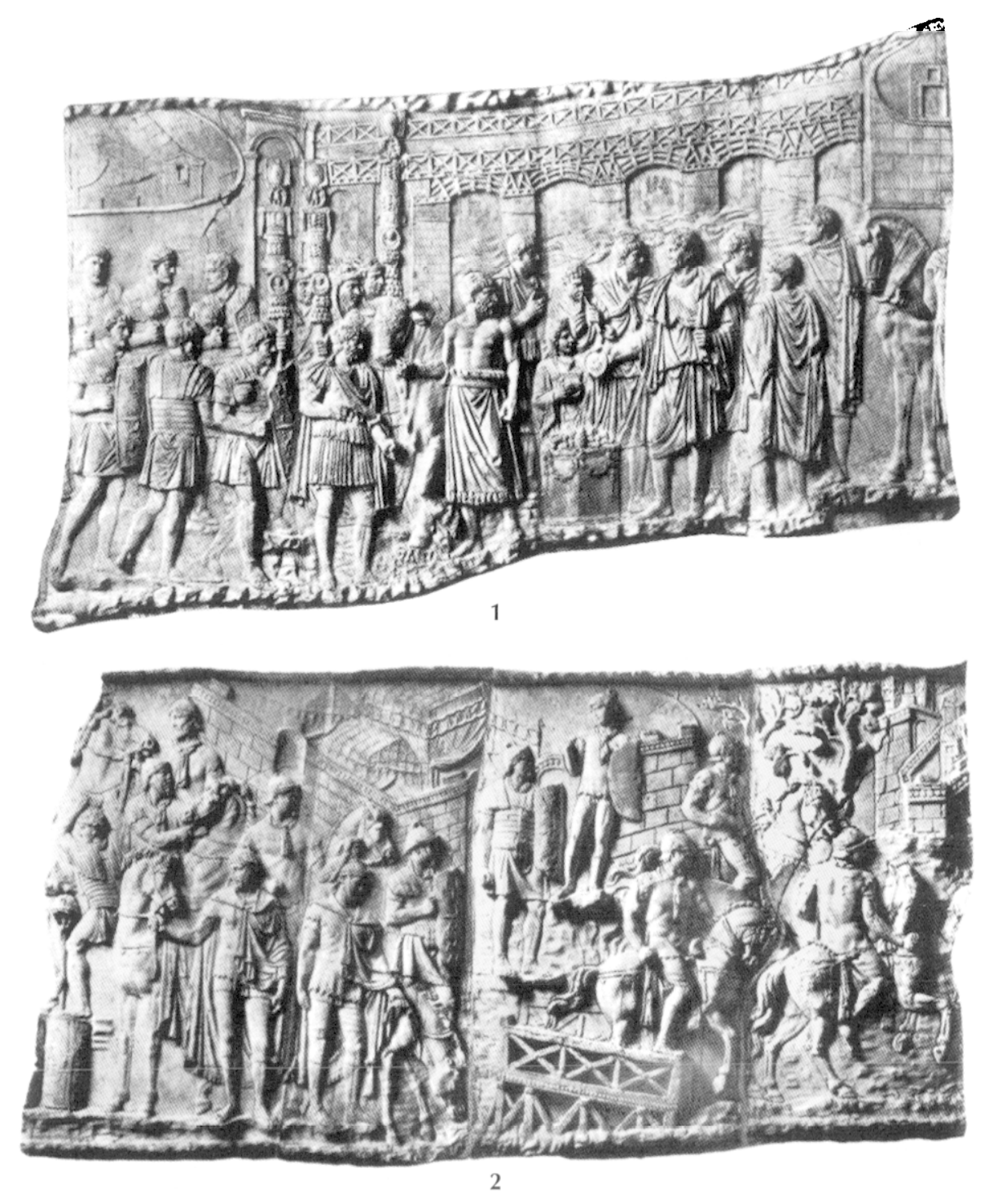

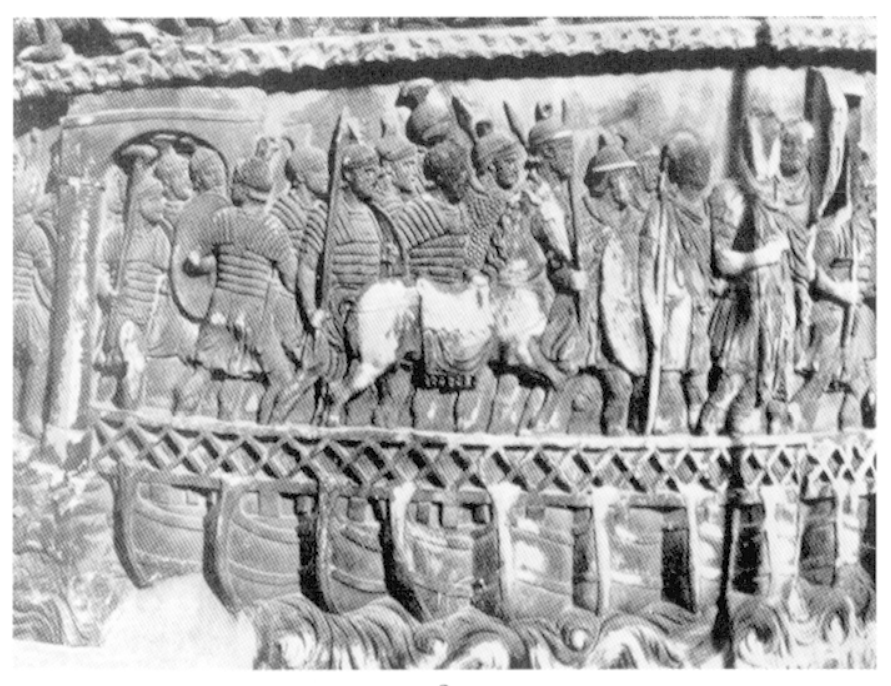

3

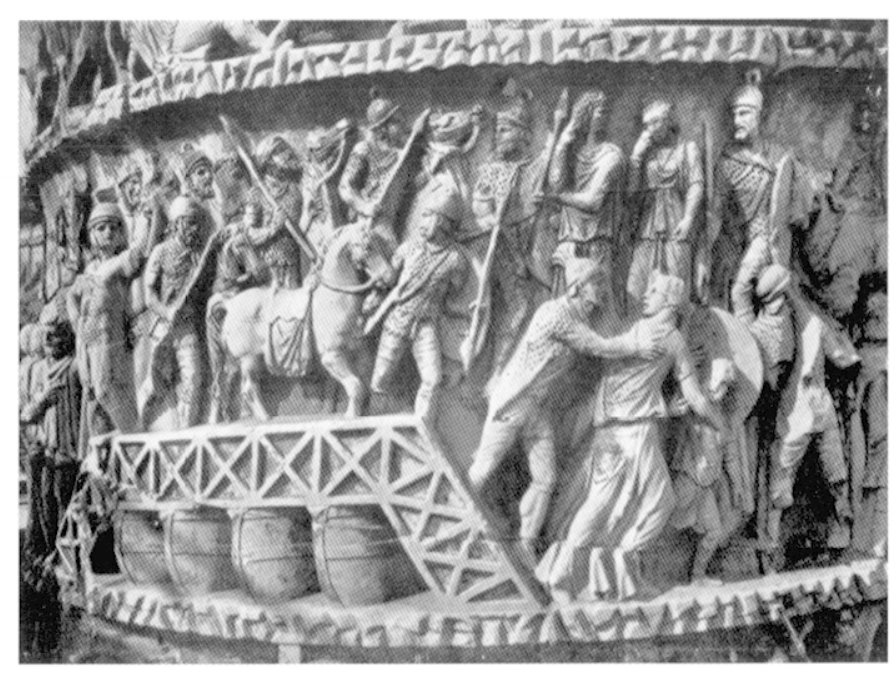

Fig. 62 - 1, scienes 98 e 99 (Cïhorius) de la colonne Trajane représentant le pont de bois construit sur le Damube par dpollodore de Damas pour les légions de Trajan. La rambarde du pont joue le rôle de poutre treillis (d'après O'Connor; 1993, fig. 117); 2, srène 21 (Cichorius) de la colonne Trajame représentant un pont de bois constitué d'une poutre treillis reposant sur des supports éux-mêmes triangulés (d'après ()'Connor; 1993, fig. 112); 3, scène de la colonne de Marr Aurèle représentant un pont de bateaux reliés par une poutre treillis (d'aprìs C. Caprino et al., 1955, fig. 9); 4, scìne de la colonne de Marc Aurèle sur laquelle est sculpté un pont de bateaux reliés par une poutre treillis qui forme une rambarde rampante à lïssue du pont (d’après (:. Capmino et al., 1955, fig. 104). 


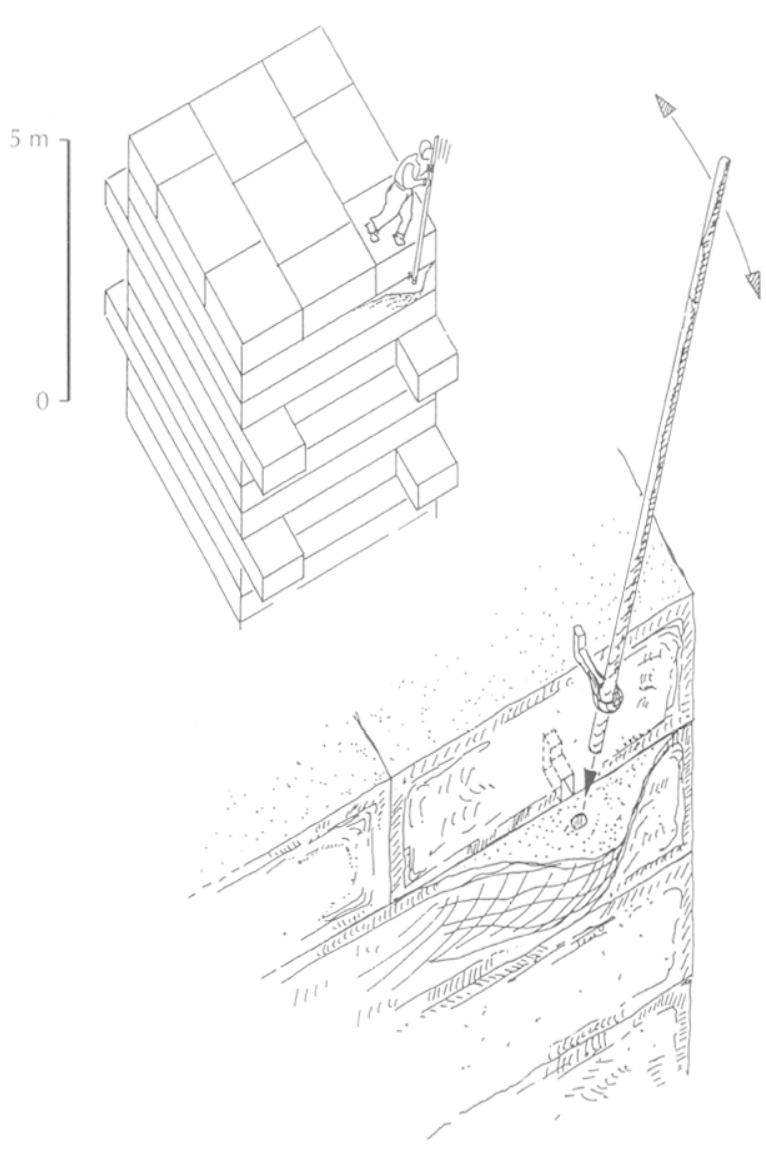

Fig. 63 - Technique de rodage du dernier bloc d'une assise d'une pile du Pont du Gard. La cavité verticale précue pour servir de logement ì la pince était ménagée dans un très gros bossage qui, par la suite, élait aballu. Parfois, même la cavité coudée creusée dans le lit de pose du bloc supérieur était aussi aménagée sur un bossage qui, après aballage, ne laissait aucume trace sur le bloc (dessin J.-I. Paillet, (XVSS).

Pour les piles du premier niveau du momument, les échafaudages ont évidemment pris appui sur le sol et nous ne voyons aucune raison de rechercher une solution plus complexe. En revanche, pour les parements des écoinçons audessus des piles et pour l'ensemble du deuxième niveau, les échafaudages traditionnels n'étaient plus acceptables, ne seraitre que pour des problèmes d'encombrement, de sécurité et de stabilité. Pour cela nous proposons une autre solution, intermédiaire entre l'échafaudage fixe et l'échafaudage volant. I.es boutisses saillantes ont probablement aussi servi à porter les platelages des échafaudages. Les boutisses sont suffisamment saillantes $(0,90 \mathrm{~m}$ environ $)$ et, en les reliant entre elles par des madriers débordants, il derient tout à fait possible d'élargir le platelage périphérique qui permettait aux bâtisseurs d'effectuer leur travail sans prendre de risques inutiles (fig. 65).

De ce fait, nous attribuons une troisième fonction à ces boutisses saillantes : elles pouraient cetre porteuses des poutres treillis, des treuils de manourre de la chère. Elles l'étaient, peut-être, aussi du platelage des échafaudages que l'on déplaçait à volonté avec la chève arrimée sur les boutisses saillantes de la pile en cours de construction.

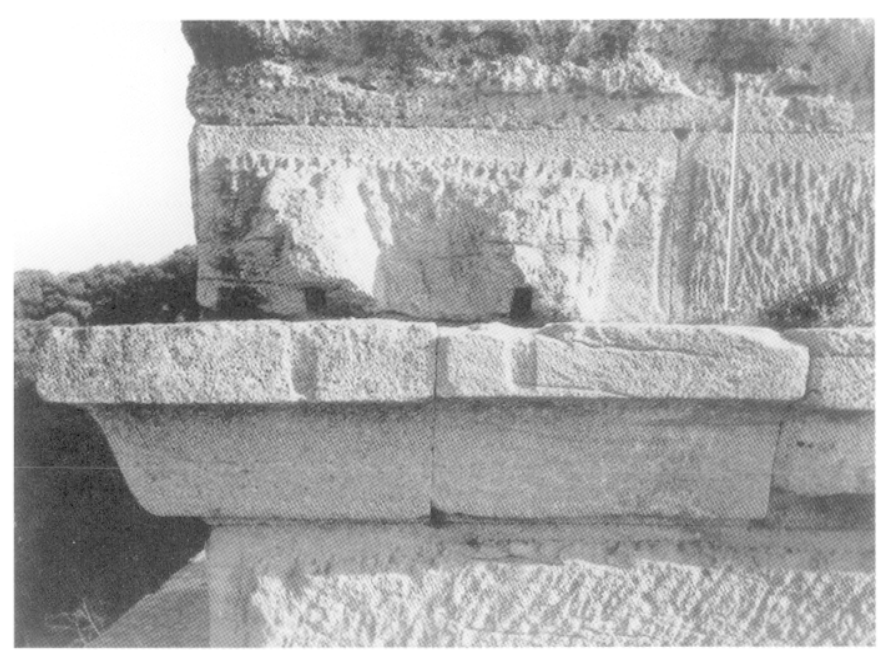

Fig. 64 - Vue de la retombée d'une arche du $3^{e}$ étage du Pont du Gard. Deux cazités coudées creusées dans des bossages d'un sommier; sur son lit de pose, sont en relation avec deux autres cavités verticales creusées dans le lit d'attente d'une imposte moulurée en corniche. Ces deux dernières ont été tronquées lors du ravalement antique de la corniche (cliché J.-L. P'aillet, (XVRS).

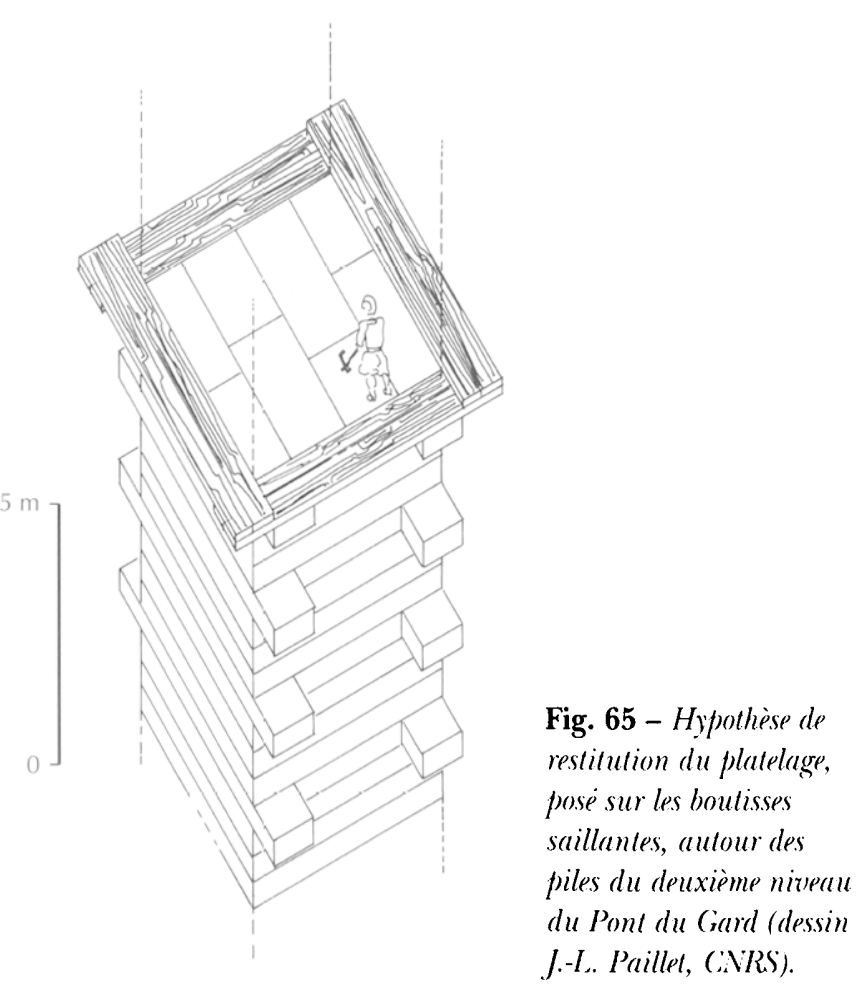

\section{LE DEUXIÈME NIVEAU}

La construction des piles et des arches du deuxième niveau du Pont du Gard suppose la mise en ocure de techniques semblables à celles utilisées pour celle du premier niveau. Toutefois, les problèmes posés par la construction à grande hauteur et lapprovisionnement du chantier imposaient des solutions adaptées à ces nouvelles contraintes. 
Trois hypothèses concernant l'approvisionnement du chantier sont possibles.

- Soit les blocs étaient charriés de la carrière à un quai aménagé dans un ancien bras mort de la rivière situé en contrebas, déposés sur des radeaux et halés par des animaux de trait remontant le cours du Gardon. Nous ne croyons guère à cette hypothèse, d'une part parce que le Gardon ne présente pas une hauteur d'eau suffisante pour que des radeaux chargés de 2 à 3 t puissent, sans racler le fond, remonter son cours et, d'autre part, parce qu'aucun chemin de halage n'est praticable en rive droite et encore moins en rive gauche. Le Gardon n'est ni le Rhône ni le Nil, et l'idée même de remonter le Gardon à la voile ou à la rame relève de la plaisanterie.

- Soit les blocs, empruntant le même parcours initial, étaient transbordés d'une rive à l'autre par un radeau faisant office de bac maintenu par des élingues. Parvenus en rive droite sur un banc de gravier quasiment horizontal, les blocs étaient chargés sur un chariot qui les acheminait au pied des piles du premier niveau du pont. Il fallait ensuite soulever les blocs depuis le sol jusqu'au tablier du premier niveau avant qu'ils ne reçoivent leurs ultimes finitions, puis les lever une nouvelle fois et les mettre en place dans l'ouvrage. Cette solution, nous l'avons vu, était valable pour les piles du premier niveau situées en rive droite ; en revanche, avec ses quatre ruptures de charge, elle paraît très complexe, coûteuse en temps et en énergie. Pour ces raisons, nous ne la retiendrons pas.

- Soit les blocs étaient directement chargés en carrière sur des charrettes qui, tirées par des boeufs, suivaient le chemin en pente douce de la rive gauche pour parvenir à la hauteur du tablier du pont du premier niveau. Arrivé en cet endroit, l'attelage pouvait poursuive sur le tablier, traverser tout l'ouvrage et décharger son fardeau aux pieds de la pile à construire. Aussi, optant pour cette dernière solution simple et ćconomique, pensons-nous que les piles du deuxième niveau ont été construites depuis la rive droite du Gardon vers la rive gauche.

Nous devons ici insister sur un détail sur lequel nous reviendrons ultérieurement. Nous supposons que, toujours en fonction d'une économie du coût du chantier, tous les voussoirs de chaque arche du deuxième niveau du pont ont été charriés et stockés en bon ordre entre les piles sur le tablier du pont du premier niveau. Ce stockage raisonné a évidemment été exécuté avant la mise en place des premières assises de la pile suivante, car après, sa construction aurait été impossible et tout voussoir oublié aurait dû être levé depuis le sol. Ce mode opératoire n'est évidemment pas sans relation avec le parti pris technique d'une préfabrication volontaire des voussoirs et de leur numérotation, lequel n'a rien à voir ni avec l'hypothèse du cintre étroit et déplacé plusieurs fois pour construire des rouleaux séparés, ni avec celle donnant une illusoire élasticité à l'ouvrage.

La construction des piles du deuxième niveau posait un problème nouveau par rapport à celle des piles de l'étage inférieur. La chève ne pouvait plus être maintenue par des cordages depuis le sol. La longucur des cordages supposés et surtout leur élasticité rendaient tout levage d'une charge importante extrêmement périlleux sinon impossible. Comme nous l'avons vu précédemment, la surface de la pile étant réduite, il était souhaitable d'éviter de l'encombrer par des treuils. Ces derniers ont dû être accrochés ou tout simplement mortaisés aux boutisses saillantes qui, en l'occurrence, faisaient office de tenons. Les treuils devaient être assemblés à un gabarit charpenté amovible qui, à chaque étape (apparemment toutes les quatre assises), pouvait être déplacé et accroché aux couples supérieurs de boutisses saillantes (fig. 60 et 61 ). Cette solution n'est possible que si des poulies folles sont installées sur l'arête horizontale supérieure des piles afin que les cordages ne se détériorent point en frottant sur les angles saillants. Pour surélever ce treuil amovible, la chèvre située sur la pile pouvait également répondre à cette autre fonction.

Une observation attentive des boutisses saillantes sur les faces latérales des piles permet de vérifier notre hypothèse. En restituant les boutisses cassées, on s'aperçoit qu'elles sont toujours disposées par couple sur un plan horizontal, qui non seulement règne sur les deux faces latérales de la pile mais aussi d'une pile à l'autre. Nous avons vu plus haut la raison de ce soin apporté à leur mise en place. Rappelons seulement que les boutisses situées au niveau des écoinçons des arches ont pu servir à supporter les poutres treillis du pont roulant indispensable à l'appareillage des voussoirs des arches. Nous attribuons donc deux fonctions essentielles à ces boutisses saillantes : d'une part, servir d'assiette aux poutres treillis porteuses du pont roulant et, d'autre part, servir de tenon pour assujettir les treuils à cage d'écureuil servant à la manœuvre de la chèvre posée sur la pile en cours de construction et au levage des blocs destinés à son édification.

Si la construction des piles du deuxième niveau a débuté en rive droite pour se terminer en rive gauche, il est probable que l'inverse se soit produit pour la mise en ceuvre des arches de ce même étage. Toujours pour faciliter l'approvisionnement du chantier, dès que la première arche a été hâtie, une équipe s'est affairée à couvrir l'extrados de l'arche et à commencer la mise en place du tablier de couvrement du deuxième niveau. Ce tablier a pu scrvir ainsi à l'approvisionnement progressif du chantier, au fur et à mesure de son avancement et de la mise en place des arches. Cette progression nous semble logique ; elle doit correspondre à la réalité.

La mise en œuvre des arches est semblable à celle des arches du niveau inférieur, à la nuance près que les treuils de manœuvre des chèvres et du pont roulant étaient assujettis à des boutisses; nous n'y reviendrons donc pas. Tous les voussoirs de chaque arche ayant été stockés en bon ordre sous le cintre, sur le tablier du pont du premier niveau (fig. 61), une chèvre pouvait les lever jusqu'au sommet de la pile où le pont roulant n'avait plus qu’à les mettre en place sur le cintre. Une "fenêtre " ménagée temporairement au travers du cintre aurait permis au pont roulant de lever directement les voussoirs et ainsi de bénéficier d'un gain de temps et d'énergie fort appréciables.

\section{LE TROISIÈME NIVEAU}

Pour la construction des piles du troisième niveau, les chariots porteurs des blocs de grand appareil bénéficiaient du tablier du pont du deuxième niveau. Il est donc logique de penser que les premières piles construites furent celles situées en rive droite. Les arches furent appareillées immédiatement après l'édification des piles à l'aide d'une chèvre qui reculait sur le tablier au fur et à mesure de la progression du chantier. 


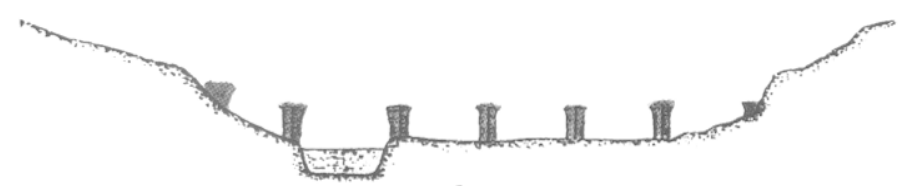

1
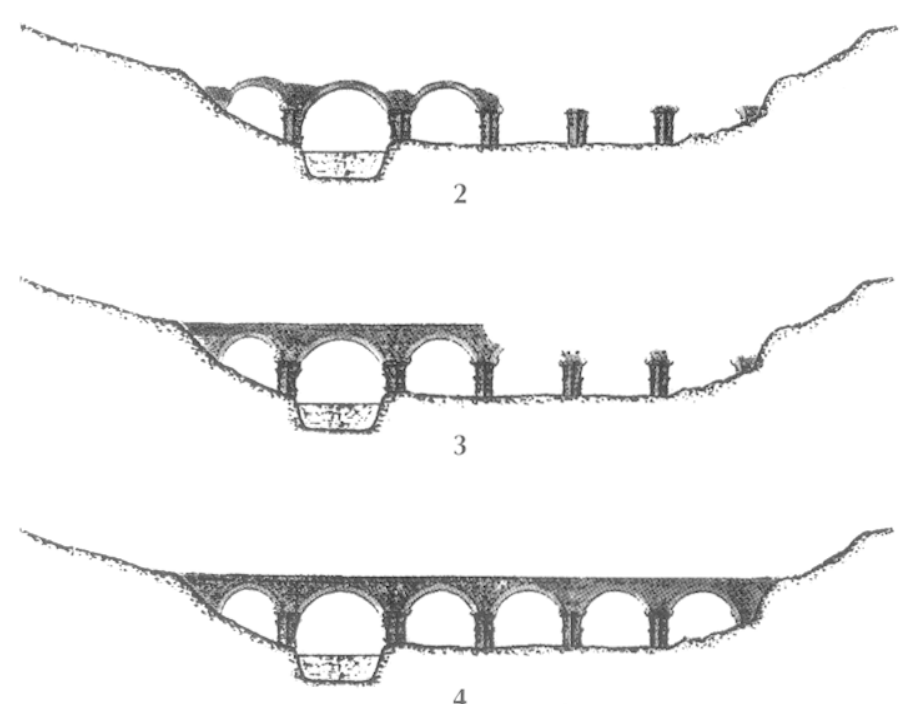

4

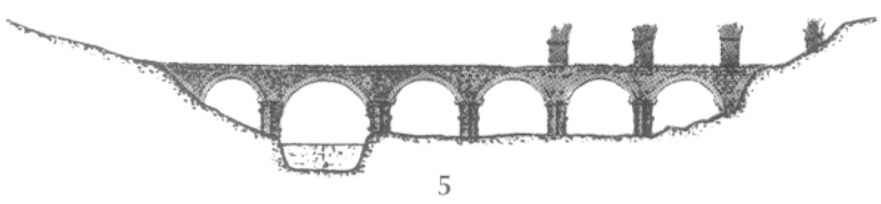

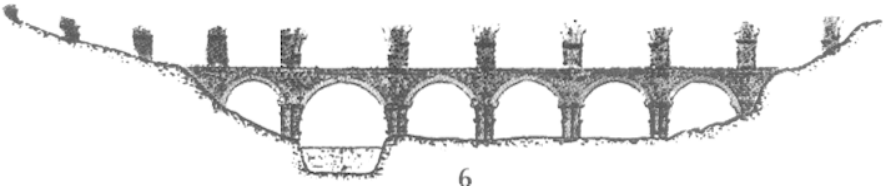

6


10

Fig. 66 - Hypothèse relative à la progression chronologique

de la construction du Pont du Gard en dix phases successives (dessin J.-L. Paillet, (NNRS).

Les grands voussoirs de ce troisième niveau étaient déchargés des chariots à l'aide d'une chèvre auxiliaire, puis déplacés sur des rondins de bois pour passer sous la grande chèvre et enfin levés et mis en place par cette dernière.

Si notre démonstration correspond à la réalité, le dernier bloc de grand appareil mis en place dans le Pont du Gard serait la clef de voûte de la première arche du troisième niveau du monument, en rive gauche du Gardon et adjacente à sa culée amont. Nous n'avons hélas aucun moyen de vérifier cette hypothèse.

Comme le troisième niveau du monument ne possède pas de tablier, nous pensons qu'après la construction des piles et des arches, les travaux se sont poursuivis à partir de la rive droite afin de pouvoir bénéficier d'un chemin de roulement sur les dalles de couverture du canal. Ainsi, une chèvre de dimensions modestes, montée sur un traîneau, pouvait approvisionner la fin du chantier en moellons de petit appareil et en mortier de chaux. Cette chèvre avançait au fur et à mesure que le canal construit recevait sa couverture de dalles.

À propos de ces dalles de couverture, il est intéressant de noter que leur épaisseur $(0,34 \mathrm{~m})$ est relativement faible par rapport à leur longueur $(3,61 \mathrm{~m})$. Les appareilleurs antiques s'en sont rendu compte et, pour éviter qu'elles ne se brisent au moment de leur mise en place, ils les ont levées à l'aide de deux louves dont les trous judicieusement écartés permettaient un levage sans aucun risque.

$$
*
$$

Le chantier du Pont du (jard, tel que nous le concevons maintenant, a certainement nécessité un nombre d'ouvriers beaucoup moins important que l'on ne l'a supposé. L'ne organisation rationnelle du chantier permet de réduire les dépenses inutiles d'énergie, le nombre d'ouvriers et la durée du chantier 55 . Le maître d'œuvre devait être présent en permanence pour indiquer la marche à suivre et éviter que le déroulement du chantier ne se passe pas comme prévu. Cette approche nous permet de proposer une chronologie de la construction du monument (fig. 66).

55. J-C. Laisné mentionne, dans son rapport de 1859, qu'en quatre années, une cinquantaine d'ourriers ont mis en ceurre plus de $2500 \mathrm{~m}^{3}$ de pierre, dont la moitié à plus de $2 \mathrm{~m}$ de haut. Nous avons calculé précédemment que le volume d'une travée movenne du Pont du Gard est de l'ordre de $3200 \mathrm{~m}^{3}$. Ces données permettent d'estimer qu'arec s00 ouvriers, le Pont du Gard a pu être construit approximativement en 5 ans. 
Notre recherche nous conduit à proposer des explications cohérentes pour les boutisses saillantes qui animent les façades latérales du monument. Leur fonction est multiple et il est probable que si elles n'ont pas été abattues, c'est en prévision d'éventuels travaux de restauration. Sans elles, il eût été impossible de réparer l'ouvrage.

La solution du pont roulant porté par des poutres en treillis est une hypothèse vraisemblable qui a pu être mise en œuvre avec les moyens techniques de l'époque. La grue tournante était connue ${ }^{56}$, mais elle aurait occupé une place

56. G. Leclli signale la découverte de roulements cylindriques, coniques et sphériques, $\mathrm{cn}$ bois dur, faisant office de roulement à bille considérable sur les piles et, de plus, sa flèche n'aurait pu excéder quelques mètres. C'est la raison pour laquelle nous avons choisi de proposer l'emploi de chèvres simples ou de mâts de charge dont le treuil, amovible, était soit amarré dans le sol soit accroché aux boutisses saillantes. Enfin, nous espérons avoir convaincu définitivement nos lecteurs que l'image du cintre étroit que l'on déplace quatre ou trois fois pour appareiller une arche du premier ou du deuxième niveau est une vue de l'esprit.

entre deux plateaux, l'un fixe, l'autre mobile. Les roulements sphériques et coniques ont pu appartenir à la base tournante d'une grue desservant le bateau ('celli, 1950, p. 191-195 et fig. 210-214). 